\title{
A three-dimensional particle method for violent sloshing under regular and irregular excitations
}

\author{
M. Luo*, C. G. Koh and W. Bai \\ Department of Civil and Environmental Engineering, National University of Singapore, Singapore 117576
}

\begin{abstract}
A three-dimensional (3D) numerical model is presented in the framework of Consistent Particle Method (CPM). The 3D gradient and Laplacian operators are derived based on Taylor series expansion, achieving good accuracy and largely alleviating the problem of spurious pressure fluctuation. An accurate boundary recognition scheme is introduced to determine the highly deformed free surface. Validated by our experimental studies of water sloshing under translational and rotational excitations, this model is shown to be robust and accurate in long time simulation of violent free surface flows which involve fluid merging and splitting. Using the validated numerical model, liquefied natural gas sloshing in a real ship under sea excitations is investigated. It is found that the sloshing waves in the beam sea sailing condition is the most violent and seriously affects the stability of ship motion. Various wave patterns under different excitations are discussed.
\end{abstract}

Keywords: particle method; three-dimensional; sloshing; rotational excitation

\section{Introduction}

It is relatively cost-effective to transport liquefied natural gas (LNG) using large ship containers. With high utilization-ratio of volume, the membrane type LNG carriers, however, are vulnerable to sloshing loads which may cause fatigue damage of the thin membrane layer, particularly in partially filled situations. To provide safe and flexible operations therefore, a better understanding of the sloshing phenomena and accurate prediction of sloshing loads in containers are very essential.

Substantial efforts have been devoted to studying liquid sloshing analytically (Faltinsen et al., 2000; Faltinsen et al., 2003; Faltinsen et al., 2005) and experimentally (Lugni et al., 2006, 2010a; Lugni et al., 2010b). With the rapid development of computer technology, numerical modelling has become increasingly feasible and many numerical algorithms have been developed to simulate sloshing problems. Among them are the mesh-based methods such as Finite Difference Method (Liu and Lin, 2008; Sriram et al., 2006; Xue and Lin, 2011), Finite Volume Method (Marsooli and Wu, 2014; Ming and Duan, 2010) and Finite Element Method (Wang and Khoo, 2005; Wu et al., 1998). These methods, however, may have some difficulties to model the large and discontinuous fluid motions which are generally involved in violent sloshing. In addition, the recognition or tracking of highly deformed free surface is also a tough issue (complicated and time-consuming) for mesh-based

\footnotetext{
* Corresponding author. Tel.: +65 8314 5868; Fax: +65 6779 1635. E-mail: 1uomin@u.nus.edu.
} 
methods although some specialized schemes have been developed such as the Volume of Fluid (Hirt and Nichols, 1981) and the level set method (Sussman et al., 1994).

In recent years, the particle methods such as Smoothed Particle Hydrodynamics (SPH), Incompressible Smoothed Particle Hydrodynamics (ISPH) and Moving Particle Semi-implicit (MPS) method have been developed and extensively used to model liquid sloshing as well as other free surface flows (Khayyer et al., 2008; Koshizuka et al., 1998; Liu et al., 2014; Monaghan, 1994; Shao et al., 2012; Shao and Lo, 2003). Because of the meshless nature, particle methods have better suitability in modelling merging and splitting of fluid and tracking of free surface. However, one of the challenging issues for these particle methods is the spurious pressure fluctuation. This is mainly caused by that the derivative approximation schemes in these methods which invoke a kernel function introduce numerical errors particularly for irregular particle distribution (Luo et al., 2015). To address this issue, a new particle method named Consistent Particle Method (CPM) has recently been proposed (Koh et al., 2012) by adopting the Generalized Finite Difference scheme (Liszka and Orkisz, 1980) to compute the spatial derivatives. Being consistent with Taylor series expansion and eliminating the use of a kernel function, CPM is fundamentally different from SPH, ISPH and MPS in terms of the derivative-approximation scheme. Due to the accurate computation of derivatives, the CPM solution of pressure history shows tremendous improvement over some other particle methods (Koh et al., 2012), without the use of artificial viscosity or smoothing technique to remove spurious fluctuation. Considering this advantage, the CPM is selected as the underlying tool of this study.

Most of the numerical works on liquid sloshing studied the sloshing phenomenon in a small tank under regular (sinusoidal) excitations in an idealized situation (Bai et al., 2015). In real applications, however, the size of the LNG container can be as big as 138,000 $\mathrm{m}^{3}$ (Lee et al., 2006) and is much larger than the tank size in the idealized condition. As pointed out by Kim et al. (2004), there are still some technical issues to apply the findings observed from small-scale sloshing to the actual tank design. Therefore, it is essential to study liquid sloshing in tank of realistic size. In addition, real LNG sloshing is excited by the ship motions which contain six degrees of freedom and are somewhat random (with many frequency components). Therefore, it is necessary to conduct a three-dimensional (3D) analysis with the consideration of the random ship motions.

Some recent works have considered the relatively more complicated sloshing problems than the idealized situation. For example, Chen and Nokes (2005) studied water sloshing under coupled (harmonic) surge, heave and pitch excitations and showed the necessity to include the coupled excitations in sloshing analysis. Wang and Khoo (2005) investigated the sloshing phenomena under horizontal random excitations. Lee et al. (2011) studied the LNG sloshing in a real tank under coupled non-harmonic surge, heave and pitch excitations. More recently, Bai et al. (2015) studied the LNG sloshing under realistic ship motions which are generated by Response Amplitude Operator (RAO), using a two-dimensional (2D) numerical model. However, very few researchers have studied 3D LNG sloshing in a real-sized tank under random sea excitations (generated by RAO), which poses greater 
challenges such as drastic increase in computational time and greater difficulty in tracking the highly deformed 3D free surface.

To address these challenges, a 3D CPM is developed in this study. The 2D CPM results have shown that it is advantageous to compute gradient and Laplacian operators based on Taylor series expansion (Koh et al., 2012); hence the computational algorithm is expanded in 3D CPM. Nevertheless, for free surface recognition, the arc method adopted in 2D CPM is not suitable for 3D problems. A 3D free surface recognition scheme is developed based on an alternative method. The 2D "spoke" method proposed in Dilts (2000) is extended herein. The accuracy of the developed numerical model is demonstrated by our experimental studies of violent water sloshing in a scaled prismatic tank under translational and rotational excitations. The wave profiles, sloshing pressures at typical positions and the wave patterns under different excitations are studied. Using the validated numerical model, LNG sloshing in a real tank under real sea excitations is investigated. The characteristics of the sloshing waves in different sailing conditions are explored.

\section{Numerical algorithms}

\subsection{Consistent Particle Method}

In CPM, the fluid domain is represented by a collection of discrete Lagrangian particles, each of which carries a fixed mass and moves under external forces mainly arising from gravity and pressure difference. The governing equations are the Navier-Stokes equations in Lagrangian form as follows

$$
\begin{gathered}
\frac{1}{\rho} \frac{D \rho}{D t}+\nabla \cdot \mathbf{v}=0 \\
\frac{D \mathbf{v}}{D t}=-\frac{1}{\rho} \nabla p+v \nabla^{2} \mathbf{v}+\mathbf{g}
\end{gathered}
$$

where $\rho$ is the density of a fluid, $\mathbf{v}$ the particle velocity vector, $p$ the fluid pressure, $v$ the kinematic viscosity of a fluid and $\mathbf{g}$ the gravity acceleration.

The above equations are solved by a predictor-corrector scheme (Koh et al., 2012; Koshizuka et al., 1998; Shao and Lo, 2003). In the predictor step, the temporary particle velocities and positions are computed by neglecting the pressure gradient term. In the corrector step, a pressure Poisson equation (PPE) is solved to enforce the incompressibility as follows

$$
\nabla^{2} p^{(k+1)}=\frac{\rho^{*}}{\Delta t^{2}} \frac{\rho^{(k+1)}-\rho^{*}}{\rho^{(k+1)}}
$$

The incompressibility condition is enforced by setting the fluid density at the current time step $\left(\rho^{(k+1)}\right)$ to be the initial value $\left(\rho_{0}\right)$. The intermediate fluid density $\left(\rho^{*}\right)$ is evaluated based on the distance-weighted average of the masses of fluid particles (including the reference particle) around the reference particle as follows 


$$
\rho_{i}^{*}=m \sum_{j} w\left(r_{i j}\right)
$$

where $m$ is the constant mass of fluid particles (identical for the particles of the same fluid), $r_{i j}$ the displacement between particle $i$ and $j$ based on intermediate positions, and $w\left(r_{i j}\right)$ a weighting function defined as follows

$$
w\left(r_{i j}\right)=\left\{\begin{array}{cl}
\frac{6 \alpha_{d}}{r_{e}^{3}} \exp \left(-\left(\frac{2 r_{i j}}{r_{e}}\right)^{2} \frac{1}{\sigma}\right), & 0<r_{i j}<r_{c} \\
\frac{3}{2 \pi r_{e}^{3}}\left(\frac{r_{e}}{r_{i j}}-1\right) & , r_{c} \leq r_{i j} \leq r_{e} \\
0 \quad, r_{i j}>r_{e}
\end{array}\right.
$$

where $r_{e}$ is the influence radius and selected to be $2.1 L_{0}\left(L_{0}\right.$ is the initial particle spacing) in the present study, $r_{c}$ is the distance of the connection point of the spline to the origin and is selected to be $0.02 r_{e}$ such that the weighting function has a very large but finite value when two particles are very close, and the coefficients $\alpha_{d}$ and $\sigma$ are determined, in a similar way (making use of the continuity condition and the partition of unity condition) as that in Koh et al. (2013) for the 2D case, to be 78.1336 and $5.34 \times 10^{-4}$ respectively. Letting the density of a fluid to be $\rho$, the constant particle mass $m$ is computed based on the initial particle distribution as follows

$$
m=\rho / \sum_{j} w\left(r_{i j}\right)
$$

Applying the derivate approximation scheme presented in Section 2.2 to the left hand side of Equation (3), a system of linear equations with sparse coefficients can be obtained and effectively solved by the generalized minimal residual (GMRES) method with incomplete LU factorization (Barrett et al., 1994). Using the solved fluid pressure, particle velocities and positions are updated. The time step should be selected to satisfy the Courant condition (Koh et al., 2012; Shao and Lo, 2003).

The above solution scheme is similar to those in MPS (Koshizuka et al., 1998) and ISPH (Shao and Lo, 2003). Nevertheless, there are two significant differences between the present method and other particle methods. The first one is the computation of the gradient and Laplacian operators and the second one is the recognition of free surface particles, as explained in the following sections.

\subsection{D derivative computation based on Taylor series expansion}

Taylor series expansion for a smooth function $f(x, y, z)$ in the vicinity of a reference particle $\left(x_{0}, y_{0}\right.$, $z_{0}$ ) can be expressed as 


$$
\begin{gathered}
f(x, y, z)=f_{0}+h f_{, x 0}+k f_{, y 0}+l f_{, z 0}+\frac{1}{2} h^{2} f_{, x x 0}+\frac{1}{2} k^{2} f_{, y y 0}+\frac{1}{2} l^{2} f_{, z z 0}+ \\
h k f_{, x y 0}+h l f_{, x z 0}+k l f_{, y z 0}+O\left(r^{3}\right)
\end{gathered}
$$

where $h=x-x_{0}, k=y-y_{0}, l=z-z_{0}, f_{0}=f\left(x_{0}, y_{0}, z_{0}\right), f_{, x 0}$ is the first order derivative of function $f$ with respect to $x$ at $\left(x_{0}, y_{0}, z_{0}\right)$ and $f_{, x y 0}$ the second-order derivative of function $f$ with respect to $x$ and $y$ at $\left(x_{0}, y_{0}, z_{0}\right)$. Writing Equation (7) for each of the neighbor particles, the following equation system can be obtained

$$
[\mathbf{A}]\{D \mathbf{f}\}-\{\mathbf{f}\}=0
$$

where $[\mathbf{A}]$ is a function of relative particle positions (i.e. $h, k$ and $l$ ), $\{\mathbf{f}\}$ a combination of the variable differences between the reference particle and its neighbor particles (i.e. $f-f_{0}$ ), and $\{D \mathbf{f}\}$ a vector including all the derivatives in Equation (7). Solving Equation (8) by a weighted-least-square approach, the first- and second-order derivatives can be directly obtained as follows

$$
\begin{gathered}
\frac{\partial p_{i}}{\partial x}=\sum_{j \neq i}\left[w _ { j } ^ { 2 } \left(a_{1} h_{j}+a_{2} k_{j}+a_{3} l_{j}+0.5 a_{4} h_{j}^{2}+0.5 a_{5} k_{j}^{2}+0.5 a_{6} l_{j}^{2}+\right.\right. \\
\left.\left.a_{7} h_{j} k_{j}+a_{8} h_{j} l_{j}+a_{9} k_{j} l_{j}\right)\left(p_{j}-p_{i}^{\prime}\right)\right]
\end{gathered}
$$

and

$$
\begin{gathered}
\frac{\partial^{2} p_{i}}{\partial x^{2}}=\sum_{j \neq i}\left[w _ { j } ^ { 2 } \left(d_{1} h_{j}+d_{2} k_{j}+d_{3} l_{j}+0.5 d_{4} h_{j}^{2}+0.5 d_{5} k_{j}^{2}+0.5 d_{6} l_{j}^{2}+\right.\right. \\
\left.\left.d_{7} h_{j} k_{j}+d_{8} h_{j} l_{j}+d_{9} k_{j} l_{j}\right)\left(p_{j}-p_{i}\right)\right]
\end{gathered}
$$

where $i$ is the reference particle and $j$ the neighbor particles in the influence domain, $w_{j}$ is the weighting function used in the weighted-least-square scheme and is adopted to be the inverse distance function (Koh et al., 2012; Liszka and Orkisz, 1980). It is noted that this weighting function is essentially different from the kernel function in SPH and ISPH (to approximate the Dirac delta function) and the weighting function in MPS (to specify the quantities diffused from the center particle to its neighbor particles). Similar to the 2D CPM (Koh et al., 2012), $p_{i}^{\prime}=\min \left(p_{j}\right)$ for $\left\{j \mid r_{i j} \leq r_{e}\right\}$ is used in Equation (9) to improve the numerical stability. Similarly, the first derivatives in the $y$ and $z$ directions can be computed by replacing $a_{s}(s=1,2 \ldots 9)$ in Equation (9) with $b_{s}$ and $c_{s}$, whereas the second derivatives in the $y$ and $z$ directions can be computed by replacing $d_{s}(s=1,2, \ldots$, 9 ) in Equation (10) with $e_{s}$ and $f_{s}$. The coefficients $a_{s}, b_{s}, c_{s}, d_{s}, e_{s}, f_{s}(s=1,2, \ldots, 9)$ can be obtained as 


$$
\left[\begin{array}{llll}
a_{1} & a_{2} & & a_{9} \\
b_{1} & b_{2} & & b_{9} \\
& & & \\
f_{1} & f_{2} & & f_{9}
\end{array}\right]=\left(\sum_{j \neq i} C_{j}\right)^{-1}
$$

where

$$
C_{j}=w_{j}^{2}\left[\begin{array}{ccccccccc}
h_{j}^{2} & h_{j} k_{j} & h_{j} l_{j} & 0.5 h_{j}^{3} & 0.5 h_{j} k_{j}^{2} & 0.5 h_{j} l_{j}^{2} & h_{j}^{2} k_{j} & h_{j}^{2} l_{j} & h_{j} k_{j} l_{j} \\
& k_{j}^{2} & k_{j} l_{j} & 0.5 h_{j}^{2} k_{j} & 0.5 k_{j}^{3} & 0.5 k_{j} l_{j}^{2} & h_{j} k_{j}^{2} & h_{j} k_{j} l_{j} & k_{j}^{2} l_{j} \\
& & l_{j}^{2} & 0.5 h_{j}^{2} l_{j} & 0.5 k_{j}^{2} l_{j} & 0.5 l_{j}^{3} & h_{j} k_{j} l_{j} & h_{j} l_{j}^{2} & k_{j} l_{j}^{2} \\
& & & 0.25 h_{j}^{4} & 0.25 h_{j}^{2} k_{j}^{2} & 0.25 h_{j}^{2} l_{j}^{2} & 0.5 h_{j}^{3} k_{j} & 0.5 h_{j}^{3} l_{j} & 0.5 h_{j}^{2} k_{j} l_{j} \\
& & & & 0.25 k_{j}^{4} & 0.25 k_{j}^{2} l_{j}^{2} & 0.5 h_{j} k_{j}^{3} & 0.5 h_{j} k_{j}^{2} l_{j} & 0.5 k_{j}^{3} l_{j} \\
& & & & & 0.25 l_{j}^{4} & 0.5 h_{j} k_{j} l_{j}^{2} & 0.5 h_{j} l_{j}^{3} & 0.5 k_{j} l_{j}^{3} \\
& & & & & & h_{j}^{2} k_{j}^{2} & h_{j}^{2} k_{j} l_{j} & h_{j} k_{j}^{2} l_{j} \\
& & & & & & & h_{j}^{2} l_{j}^{2} & h_{j} k_{j} l_{j}^{2} \\
& & & & & & & & k_{j}^{2} l_{j}^{2}
\end{array}\right]
$$

The matrix size in Equation (11) is $9 \times 9$. The last three rows in the left hand side correspond to the cross partial derivative terms. They are not shown here because they are not needed in the computation of Laplacian operator of pressure. The coefficient matrix in the right hand side of Equation (11) is symmetric positive definite and hence this equation can be effectively solved using the Cholesky decomposition method. It is noted that more than $90 \%$ of the computational time is used to solve the linear equation system generated by discretizing Equation (3). Hence, the computational time to solve the local matrixes, i.e. Equation (11), is negligible. In addition, since CPM largely alleviated the spurious pressure fluctuation that exists in some other particle methods, an iterative solver would converge fast when solving the linear equation. Therefore, the computational efficiency of CPM should be at least comparable to those of MPS and ISPH (both of which need to solve a PPE).

\subsection{D Free surface recognition}

In 2D CPM, the free surface particles are recognized by the arc method (Dilts, 2000; Koh et al., 2012). The basic idea is that, if the arcs of a circle around a reference particle are fully covered by the circles of its neighbor particles, this reference particle is not a free surface particle. The $3 \mathrm{D}$ version of the arc method requires the checking of the intersection area of two spheres (instead of circles), which is complicated and inefficient (Haque and Dilts, 2007). In contrast, the spoke method for 2D problems (Dilts, 2000) can be extended to 3D problems more easily and is thus adopted in the present study. The key idea is to scan the sphere of radius $R$ around a reference particle (see Fig. 1). If any region of the surface of this sphere is not covered by the spheres (of the same radius) of its neighbor particles, this particle is recognized as a free surface particle. More specifically, circles of longitude (red circles in Fig. 1) are determined on the surface of the sphere with angle interval $\alpha$. On each circle of longitude, points are uniformly generated (small empty circles in Fig. 1) with angle interval $\beta$. If all 
the generated points are covered by the spheres of any neighbor particle, this reference particle is treated as an inner particle. Otherwise, it is a free surface particle. Based on our parametric studies, the use of $R=1.3 L_{0}$ ( $L_{0}$ is the initial particle spacing), $\alpha=22.5^{\circ}$ and $\beta=22.5^{\circ}$ gives accurate and efficient recognition of free surface particles and is used in all the cases of this study. Purely based on the relative positions of particles, the proposed scheme is more accurate in identifying the free surface than the density threshold approaches. In addition, the recommended parameters are general and can be used in other 3D simulation of free surface flows.

\section{Water sloshing under translational excitation}

The developed 3D CPM is first used to study water sloshing in a prismatic tank under translational excitation. For validation purpose, experiments are conducted on a uni-axial shake table (Fig. 2), which can generate translational motion governed by $A(t) \sin (\omega t+\pi)$, where $A(t)$ and $\omega$ are the amplitude and frequency of the excitation respectively. To avoid a sudden jerk on fluid caused by the non-zero initial velocity of the shake table, the excitation with a linear ramping function $A_{0} t / t_{r}$ is used, where $t_{r}=10 \mathrm{~s}$ is the ramping time and $A_{0}=0.005 \mathrm{~m}$ is the steady-state amplitude of the excitation. Scaled down geometrically from a real LNG membrane tank (Lloyd's Register, 2008), the tank used in experiments is made of Plexiglas of thickness $10 \mathrm{~mm}$ and with its size shown in Fig. 3, where $L$ is the water length, $d$ the water depth, $H$ the tank height and $B$ the tank breadth. The tank is bolted to the shake table. To obtain 3D sloshing waves, the longitudinal axis of the tank is not in line with that of the shake table but with an oblique angle $\theta$ (selected to be $45^{\circ}$ in the present study) as shown in Fig. 4. This setup is used to investigate 3D sloshing in membrane LNG tanks. In real LNG sloshing cases, sloshing is severe in mid-filling levels from $10 \% L_{\mathrm{T}}$ (the length of tank) to $70 \% \mathrm{H}$ (Lloyd's Register, 2008). Therefore, the filling ratio of $d / H=0.5$ is adopted in this section. Based on linear wave theory, the fundamental natural frequencies for the sloshing system in the directions of length and breadth ( $L$ and $B$ in Fig. 3 ) are $\omega_{L 0}=6.618 \mathrm{rad} / \mathrm{s}$ and $\omega_{B 0}=8.364 \mathrm{rad} / \mathrm{s}$, respectively. The dynamic pressures are measured by gauge pressure sensors (WIKA S-10) at $\mathrm{P}_{1}$ (middle point along the width) and $\mathrm{P}_{2}$ (near corner) on the left wall of the tank (see Fig. 3 and Fig. 4), which are both 100 $\mathrm{mm}$ vertically from the bottom of the tank and $20 \mathrm{~mm}$ and $200 \mathrm{~mm}$ horizontally from the front wall respectively. The dynamic sloshing wave motions are captured by a video camera. In the numerical simulation, an initial particle spacing of $0.008 \mathrm{~m}$ (129,826 particles in total) and a fixed time step of $0.001 \mathrm{~s}$ are adopted (these parameters are also used in the other cases of this section). The computational time is about 3.6 hours per 1000 time steps on a personal computer with Intel(R) Core 17-2600. The tank walls are modeled as rigid and fixed particles (relative to the tank motion), and the moving container is simulated by updating the positions of the wall particles in accordance with the tank motion. This wall treatment approach is used in all the numerical examples of this study. 
First, the length-directional resonance scenario with excitation frequency equal to $\omega_{L 0}$ (i.e. the fundamental natural frequency in the length direction) is studied. Fig. 5 shows that the simulated sloshing waves at various time instants are in good agreement with the corresponding experimental results. This demonstrates the accuracy of the 3D CPM. It is noted that although the excitation has an oblique angle with the axis in the length direction ( $x$ axis) of the tank, the sloshing wave behaves like a nearly-planar wave in the plane of the length direction ( $x-z$ plane). The reason is that the excitation frequency results waves in resonance in the length direction, which dominates waves in the breadth direction. The wave begins to touch the chamfer knuckle of the prismatic tank at about $9.9 \mathrm{~s}$. In the subsequent cycles, breaking waves appear as shown in the snapshots of $t=13.72 \mathrm{~s}$ and $14.675 \mathrm{~s}$. The accuracy of CPM is further demonstrated by Fig. 6, in which the simulated sloshing pressure at point $\mathrm{P}_{1}$ is in fairly good agreement with the experimental result in terms of pressure amplitude and phase. The relative error of the largest pressure peak is only $6.5 \%$.

The wave pattern in the prismatic tank is investigated by studying the elevations of wave run-up at the middle of the left wall (E1 in Fig. 3) and front wall (E2 in Fig. 3). Fig. 7a shows the results of the length-directional resonance case. The wave run-up at E1 keeps increasing in the studied period and reaches about $0.14 \mathrm{~m}$ at $t=15 \mathrm{~s}$, while the wave elevation at E2 is very small. The very small wave elevation at E2 arises from higher modes of the sloshing wave (Faltinsen et al., 2003). For better illustration, the wave elevations at E1 and E2 are compared in the E1-E2 plot in Fig. 7a, which shows nearly a horizontal line. It means that the wave in this case is a nearly planar wave in the $x-z$ plane, which is termed the length-dominant wave. Conversely, when the excitation frequency is equal to the fundamental natural frequency in the breadth direction (i.e. $\omega=\omega_{B 0}$ ), the sloshing wave behaves as a breadth-dominant wave as shown in Fig. 7b. These phenomena are different from those in a square (or nearly square) tank for which Faltinsen and Timokha (2009) observed planar wave only when the excitation frequency was not in the vicinity of the fundamental natural frequency. This is because in a square (or nearly square) tank, the natural frequencies in the length and breadth directions are very close. Therefore, the resonant excitation frequency induces violent waves in both directions, thereby manifesting complex 3D waves.

In addition to the length and breadth resonance cases, an excitation frequency $(7.445 \mathrm{rad} / \mathrm{s})$ between $\omega_{L 0}$ and $\omega_{B 0}$ is studied. As shown in Fig. 7c, the wave elevations at E1 and E2 both increase with time and reach the steady state after about ten cycles. The superposition of the waves along the two directions leads to the nearly-diagonal wave which has also been reported by Faltinsen and Timokha (2009). This wave pattern can be more clearly seen from the E1-E2 plot, which looks like an ellipse whose major axis represents the direction in which waves travel. 


\section{Water sloshing under rotational excitation}

\subsection{Experimental set-up}

Next, the CPM is used to simulate more complicated sloshing phenomena under rotational excitations. Experimental studies are conducted on a rotational simulator as shown in Fig. 8. The rotations about the $x$ and $y$ axes are respectively defined as pitch and roll as shown in Fig. 9. The same tank as that in Section 3 is used and fixed to the platform of the rotational simulator with the center of the tank bottom coinciding with the center of the top of the platform (point O' in Fig. 9). Based on our parametric studies, the filling depth of $d / H=0.3$ generates large sloshing pressure and is adopted as the case study in this section. Estimated by linear wave theory, the fundamental natural frequencies for the sloshing system in the roll and pitch directions are $5.598 \mathrm{rad} / \mathrm{s}$ and $7.471 \mathrm{rad} / \mathrm{s} \mathrm{respectively.}$ Dynamic pressures at $\mathrm{P}_{1}$ and $\mathrm{P}_{2}$ are measured by gauge pressure sensors and the sloshing wave motions are captured by a video camera.

The rotational simulator is schematically shown in Fig. 9. Two alternating-current motors generate rotations of the rigid platform about the $x$ and $y$ axes with point $\mathrm{O}$ as the pivot. A rigid connector O-O' is perpendicularly fixed to the platform and with length $149.49 \mathrm{~mm}$. To obtain the actual roll and pitch motions of the simulator, the vertical displacements at two diagonally opposite points on the simulator platform are measured by two vertically positioned displacement transducers $\left(\mathrm{DT}_{1}\right.$ and $\mathrm{DT}_{2}$ in Fig. 8 and Fig. 9). Both displacement and pressure signals are recorded by a digital oscilloscope.

\subsection{Case 1: Sloshing under resonant excitation}

In Case 1, the excitation frequencies of roll and pitch rotations are set to be the same as the corresponding natural frequencies, i.e. $5.598 \mathrm{rad} / \mathrm{s}$ and $7.471 \mathrm{rad} / \mathrm{s}$ respectively. The measured rotations for the two directions are as shown in Fig. 10. The pitch motion is not sinusoidal because of the control precision of the simulator. However, this does not influence the results since the real motions are used as the input of numerical simulations. In the numerical model, an initial particle spacing of $0.008 \mathrm{~m}(116,361$ particles in total) and fixed time step of $0.0005 \mathrm{~s}$ are used (these parameters are also used in Case 2 in Section 4.3). The wave profiles at different time instants are presented in Fig. 11, showing generally good agreement between the numerical and experimental results. In the beginning, a swirling wave that rotates anticlockwise (viewed from top) is generated in the tank. Wave breaking occurs on the left wall of the tank at about $1.96 \mathrm{~s}$. After $t=2.40 \mathrm{~s}$, the swirling wave changes its direction to be clockwise which can be seen from the snapshots progressively from $2.40 \mathrm{~s}$ to $2.86 \mathrm{~s}$. From about $5.26 \mathrm{~s}$ to $5.80 \mathrm{~s}$, the swirling wave becomes anticlockwise again. The swirling wave can be explained by the superposition of the wave components in the length and breadth directions as

$$
A(x, y)=A_{x} \cdot f_{1,0}(x, y) \cdot \sin \left(\omega_{x} t+\varphi_{x}\right)+A_{y} \cdot f_{0,1}(x, y) \cdot \sin \left(\omega_{y} t+\varphi_{y}\right)
$$


where $f_{1,0}(x, y), A_{x}, \omega_{x}$ and $\varphi_{x}$ are respectively the first modal shape of the wave, amplitude of wave elevation, and the frequency and phase angle of the wave in the length direction, whereas $f_{0,1}(x, y)$, $A_{y}, \omega_{y}$ and $\varphi_{y}$ are those parameters in the breadth direction. Because $\omega_{x}$ and $\omega_{y}$ are different, the swirling wave changes its rotary direction cyclically (with a "beating" frequency of $\left|\omega_{x}-\omega_{y}\right|$ ), which can also be seen from the pressure result presented in Fig. 12. This observation is different from the sloshing phenomenon under 1-degree-of- freedom translational excitation (Faltinsen et al., 2005), in which the rotary direction of swirling is dependent on the initial perturbation of wave and theoretically does not change.

A practical significance is that swirling waves generate large impact force near the corner of the tank as presented in Fig. 12, which shows the sloshing pressures at $\mathrm{P}_{1}$ and $\mathrm{P}_{2}$. Due to the impact of swirling wave at the tank corner, the impact pressure at $\mathrm{P}_{2}$ is larger than that at $\mathrm{P}_{1}$ at the middle of the wall. 3D-CPM captures this phenomenon with the numerical results in generally good agreement with the experimental results. In particular, the large impact peaks at the tank corner are accurately predicted by the CPM as can be seen from the enlarged view of Fig. 12. The relative difference between the CPM and experimental results is only 3.9\% for the largest pressure peak. Some minor discrepancies exist between the numerical and experimental results of pressure at the reversals of swirling direction, the reason for which is as follows. The changes of wave swirling direction correspond to the changes of rotational direction of the simulator, which implies larger rotational accelerations. Since the tip of the displacement transducer is attached to the top of the platform, larger friction force exists between the transducer and the platform when rotational acceleration of the platform is larger. The larger friction force at the tip of the displacement transducer further induces larger errors in the measured rotational angles. Since the measured rotational angles of the rotational simulator are used as the excitation input of CPM simulation, some errors are brought into the numerical simulation. Although measures are taken to minimise the friction force between the displacement transducer and the platform, it is hard to remove this kind of experimental error.

\subsection{Case 2: Sloshing under random excitations}

In real sea conditions, the excitation that causes sloshing is generally irregular and contains many frequency components. In Case 2, sloshing under random excitations is studied. Fig. 13 shows the roll and pitch motions generated using the approach described in Section 5.2 and a scaled RAO. The simulated wave profiles at typical time instants are presented in Fig. 14, which show good agreement with the experimental results. In the presented results, the sloshing wave is predominantly rolldominant. At about $t=5.84 \mathrm{~s}$, violent wave breaking occurs because the roll angle is large. For the sloshing pressures at $\mathrm{P}_{1}$ and $\mathrm{P}_{2}$, Fig. 15 shows generally good agreement between the CPM and experimental results even for a long-time simulation of $35 \mathrm{~s}$. The relative errors for the largest pressure peaks at $\mathrm{P}_{1}$ and $\mathrm{P}_{2}$ are $8.1 \%$ and $13.1 \%$ respectively. This demonstrates the capability of 3D 
CPM, which is then used to study LNG sloshing in a real LNG ship under real sea excitations, as presented in the next section.

\section{LNG sloshing in ship under real sea excitations}

\subsection{Ship and tank parameters}

Fig. 16 shows the schematic diagram (top view) of a LNG ship and the definition of the coordinate system. The $x$ axis is in the forward surge direction, the $y$ axis in the sway direction toward the port side, and the $z$ axis in the upward direction. The origin of the coordinates is at the center of gravity of the ship. One of the cargo tanks at the forward part of the vessel is used in this study, as shown in Fig. 17. The encounter angle and sailing condition are illustrated in Fig. 16. Three cases are considered: 90 degrees for Beam Sea (from starboard to port side), 180 degrees for Head Sea and 135 degrees for Bow Sea. For the sea keeping analysis, the wave frequency range is 0.2 to $2.0 \mathrm{rad} / \mathrm{s}$ with an increment of $0.02 \mathrm{rad} / \mathrm{s}$. The density and dynamic viscosity of LNG are $425 \mathrm{~kg} / \mathrm{m}^{3}$ and $10^{-4} \mathrm{~Pa} \cdot \mathrm{s}$ respectively.

\subsection{Ship motion in seaway}

Random sea wave motion can be modelled in a stochastic way by the sum of a large number of independent regular contributions with random phases. Accordingly, the ship motion of the $i$-th degree of freedom (e.g. roll, pitch, etc.) can be approximated as (Perez and Blanke, 2002)

$$
\eta_{i}(t)=\sum_{k=1}^{N} \bar{\eta}_{i k} \cos \left(\omega_{e k} t+\theta_{k}\right)
$$

where $\theta_{k}, \omega_{e k}$ and $\bar{\eta}_{i k}$ are the random phase angle, the encounter frequency and the amplitude of the $k$-th component, respectively. Given the $k$-th wave frequency $\left(\omega_{k}\right)$, the encounter frequency can be computed as

$$
\omega_{e k}=\omega_{k}-\frac{\omega_{k}^{2} U_{0} \cos \chi}{g}
$$

where $U_{0}$ is the design speed of a ship, $\chi$ the wave encounter angle and $g$ the gravity acceleration.

The amplitude of the $k$-th component can be computed as

$$
\bar{\eta}_{i k}=\sqrt{2\left|R_{\eta_{i} \zeta}\left(\omega_{k}, \chi, U\right)\right|^{2} \int_{\omega_{1 k}}^{\omega_{2 k}} S(\omega) d \omega}
$$

where $\omega_{1 k}=\omega_{k}-\Delta_{1 k}, \omega_{2 k}=\omega_{k}+\Delta_{2 k}, \Delta_{1 k}=\left(\omega_{k}-\omega_{k-1}\right) / 2, \Delta_{2 k}=\left(\omega_{k+1}-\omega_{k}\right) / 2, R_{\eta_{i}}\left(\omega_{k}, \chi, U\right)$ is the RAO which relates ship motion and wave height to wave frequency and $S(\omega)$ the wave spectrum. The RAOs for six degrees of freedom are based on a fully loaded LNG ship (introduced in Section 5.1) with the design speed 20.15 knots $(10.366 \mathrm{~m} / \mathrm{s})$. The Modified Pierson-Moskowits (MPM) wave spectrum is adopted as follows 


$$
S(\omega)=\frac{4 \pi^{3} h_{1 / 3}^{2}}{\omega^{5} T^{4}} \exp \left(\frac{-16 \pi^{3}}{\omega^{4} T^{4}}\right)
$$

where $h_{1 / 3}$ is the significant wave height and $T$ the dominant wave period. These two parameters are adopted to be $4 \mathrm{~m}$ and $5 \pi \mathrm{s}$ respectively in the following cases.

\subsection{LNG sloshing in ship under sea excitations}

When sailing on the sea, a LNG ship may encounter different heading conditions, in which the pattern and severity of sloshing waves can be quite different. Therefore, the characteristics of sloshing waves in three typical sailing conditions are studied, i.e. Head Sea, Beam Sea and Bow Sea 135 degrees. Sloshing waves are the most violent for middle filling levels, and hence the half-filling case $(d / H=0.5)$ is considered in this section. Since a LNG ship normally has several LNG tanks, letting one of the LNG tanks to be half-filling will not considerably change the RAOs of the ship. Therefore, it is acceptable to use the RAO data generated based on a fully loaded condition to study half-filling LNG sloshing. In the following numerical simulations, an initial particle spacing of $0.5 \mathrm{~m}(256,778$ particles in total) and a fixed time step of $0.01 \mathrm{~s}$ are used. The computational time is about 16.5 hours per 1000 time steps on a personal computer with Intel(R) Core i7-2600.

\subsubsection{LNG sloshing in Head Sea}

When a ship is sailing in Head Sea, the motions in sway, roll and yaw are generally small and hence only the surge, heave and pitch excitations as shown in Fig. 18 are considered. A 3D-CPM simulation is conducted up to $200 \mathrm{~s}$ and the snapshots of sloshing waves at typical time instants are presented in Fig. 19. Standing wave in the $x-z$ plane is observed. In the period of $180 \mathrm{~s}$ to $200 \mathrm{~s}$, sloshing waves with relatively large amplitude are observed because the pitch motion is large.

\subsubsection{LNG sloshing in Beam Sea}

Another typical case to consider is Beam Sea, for which the ship motions in sway, heave and roll are dominant. Therefore, only these three excitations as shown in Fig. 20 are studied and the numerical wave profiles are presented in Fig. 21. In this condition, the wave motions are much more violent than those in the Head Sea condition. The LNG motion exhibits progressive wave propagating to and fro between starboard and port. Breaking waves are observed which generate large impact forces on the tank wall and ceiling. The violent sloshing waves also cause the shift of the center of gravity of the container, which may seriously affect the ship control under sail.

\subsubsection{LNG sloshing in Bow Sea 135 degrees}

When a ship sails in Bow Sea 135 degrees (Fig. 22), the ship motions with six degrees of freedom should be considered. The wave profiles at typical instants are shown in Fig. 23. The sloshing wave is complex with frequent changes of wave pattern and is generally much more violent than the Head Sea 
case. In the first $20 \mathrm{~s}$, the sloshing wave primarily travels in the diagonal direction. From $20 \mathrm{~s}$ to $40 \mathrm{~s}$, the sloshing wave behaves as a progressive wave moving between starboard and port because the roll motion is large at this stage. From $100 \mathrm{~s}$ to $120 \mathrm{~s}$, irregular 3D waves are observed. Form $140 \mathrm{~s}$ to 180 $\mathrm{s}$ when both heave and roll motions are large, violent breaking waves are observed that would generate large impact force on tank walls and possibly even cause the stability problem of ship motion.

\section{Conclusions}

In this study, the recently developed CPM is extended to simulate 3D sloshing waves. The $3 \mathrm{D}$ spatial derivatives are computed in a way consistent with Taylor series expansion, producing good accuracy even for irregular particle distributions. To define the free surface, the arc method used in 2D problems is too complicated and hence a new scheme is developed based on the spoke method. The 3D CPM is used to simulate the water sloshing in a scaled tank under translational and rotational (regular and random) excitations. The various wave patterns, including length-, width- and diagonaldominant waves and swirling waves, which can only be observed in the 3D study. These complex wave motions and the sloshing pressures predicted by the 3D CPM are in fairly good agreement with the experimental results.

Using the validated numerical model, LNG sloshing in a real ship under sea excitations is studied for three typical sailing conditions. In this case study, the sloshing wave in the Head Sea condition is not as critical as the other two cases. In the Beam Sea condition and other general conditions such as Bow Sea 135 degrees considered where the roll motion is large, very violent sloshing waves can be induced which not only generate large impact force on tank wall but may also shift the center of gravity of the tank significantly to affect the control of ship motion.

\section{Acknowledgement}

The authors appreciate the funding support of the Maritime and Port Authority of Singapore (Grant WBS No. R-302-000-012-490) and the technical support of Singapore Offshore Technology Center of American Bureau of Shipping.

\section{References}

Bai, W., Liu, X., Koh, C.G., 2015. Numerical study of violent LNG sloshing induced by realistic ship motions using level set method. Ocean Engineering 97, 100-113.

Barrett, R., Berry, M.W., Chan, T.F., Demmel, J., Donato, J., Dongarra, J., Eijkhout, V., Pozo, R., Romine, C., Vorst, H.v.d., 1994. Templates for the Solution of Linear Systems: Building Blocks for Iterative Methods.

Chen, B.-F., Nokes, R., 2005. Time-independent finite difference analysis of fully non-linear and viscous fluid sloshing in a rectangular tank. Journal of Computational Physics 209, 47-81. 
Dilts, G.A., 2000. Moving least-squares particle hydrodynamics II: conservation and boundaries. International Journal for Numerical Methods in Engineering 48, 1503-1524.

Faltinsen, O.M., Rognebakke, O.F., Lukovsky, I.A., Timokha, A.N., 2000. Multidimensional modal analysis of nonlinear sloshing in a rectangular tank with finite water depth. Journal of Fluid Mechanics 407, 201-234.

Faltinsen, O.M., Rognebakke, O.F., Timokha, A.N., 2003. Resonant three-dimensional nonlinear sloshing in a square-base basin. Journal of Fluid Mechanics 487, 1-42.

Faltinsen, O.M., Rognebakke, O.F., Timokha, A.N., 2005. Classification of three-dimensional nonlinear sloshing in a square-base tank with finite depth. Journal of Fluids and Structures 20, 81-103.

Faltinsen, O.M., Timokha, A.N., 2009. Sloshing. Cambridge University Press, New York.

Haque, A., Dilts, G.A., 2007. Three-dimensional boundary detection for particle methods. Journal of Computational Physics 226, 1710-1730.

Hirt, C.W., Nichols, B.D., 1981. Volume of fluid (VOF) method for the dynamics of free boundaries. Journal of Computational Physics 39, 201-225.

Khayyer, A., Gotoh, H., Shao, S.D., 2008. Corrected incompressible SPH method for accurate watersurface tracking in breaking waves. Coastal Engineering 55, 236-250.

Kim, Y., Shin, Y.-S., Lee, K.H., 2004. Numerical study on slosh-induced impact pressures on threedimensional prismatic tanks. Applied Ocean Research 26, 213-226.

Koh, C.G., Gao, M., Luo, C., 2012. A new particle method for simulation of incompressible free surface flow problems. International Journal for Numerical Methods in Engineering 89, 1582-1604.

Koh, C.G., Luo, M., Gao, M., Bai, W., 2013. Modelling of liquid sloshing with constrained floating baffle. Computers \& Structures 122, 270-279.

Koshizuka, S., Nobe, A., Oka, Y., 1998. Numerical analysis of breaking waves using the moving particle semi-implicit method. International Journal for Numerical Methods in Fluids 26, 751-769.

Lee, S.-H., Lee, Y.-G., Jeong, K.-L., 2011. Numerical simulation of three-dimensional sloshing phenomena using a finite difference method with marker-density scheme. Ocean Engineering 38, 206225. 
Lee, Y.B., Lee, J.M., Kim, Y.S., Jung, J.H., Jung, D.W., Kwon, S.H., 2006. An Experimental Study of Impulsive Sloshing Load Acting on LNGC Tank, International Offshore and Polar Engineering Conference (16th), San Francisco, California, USA.

Liszka, T., Orkisz, J., 1980. The finite difference method at arbitrary irregular grids and its application in applied mechanics. Computers \& Structures 11, 83-95.

Liu, D., Lin, P., 2008. A numerical study of three-dimensional liquid sloshing in tanks. Journal of Computational Physics 227, 3921-3939.

Liu, X., Lin, P., Shao, S., 2014. An ISPH simulation of coupled structure interaction with free surface flows. Journal of Fluids and Structures 48, 46-61.

Lloyd's Register, 2008. Guidance on the operation of membrane LNG ships to reduce the risk of damage due to sloshing. Lloyd's Register, London.

Lugni, C., Brocchini, M., Faltinsen, O.M., 2006. Wave impact loads: The role of the flip-through. Physics of Fluids 18, 122101.

Lugni, C., Brocchini, M., Faltinsen, O.M., 2010a. Evolution of the air cavity during a depressurized wave impact. II. The dynamic field. Physics of Fluids 22, 056102.

Lugni, C., Miozzi, M., Brocchini, M., Faltinsen, O.M., 2010b. Evolution of the air cavity during a depressurized wave impact. I. The kinematic flow field. Physics of Fluids 22, 056101.

Luo, M., Koh, C.G., Gao, M., Bai, W., 2015. A particle method for two-phase flows with large density difference. International Journal for Numerical Methods in Engineering 103, 235-255.

Marsooli, R., Wu, W., 2014. 3-D finite-volume model of dam-break flow over uneven beds based on VOF method. Advances in Water Resources 70, 104-117.

Ming, P.-j., Duan, W.-y., 2010. Numerical simulation of sloshing in rectangular tank with VOF based on unstructured grids. Journal of Hydrodynamics, Ser. B 22, 856-864.

Monaghan, J.J., 1994. Simulating free surface flows with SPH. Journal of Computational Physics 110, 399-406.

Perez, T., Blanke, M., 2002. Simulation of ship motion in seaway. Department of Electrical and Computer Engineering, The University of Newcastle, Australia.

Shao, J.R., Li, H.Q., Liu, G.R., Liu, M.B., 2012. An improved SPH method for modeling liquid sloshing dynamics. Computers \& Structures 100-101, 18-26. 
Shao, S., Lo, E.Y.M., 2003. Incompressible SPH method for simulating Newtonian and nonNewtonian flows with a free surface. Advances in Water Resources 26, 787-800.

Sriram, V., Sannasiraj, S.A., Sundar, V., 2006. Numerical simulation of 2D sloshing waves due to horizontal and vertical random excitation. Applied Ocean Research 28, 19-32.

Sussman, M., Smereka, P., Osher, S., 1994. A level set approach for computing solutions to incompressible two-phase flow. Journal of Computational Physics 114, 146-159.

Wang, C.Z., Khoo, B.C., 2005. Finite element analysis of two-dimensional nonlinear sloshing problems in random excitations. Ocean Engineering 32, 107-133.

Wu, G.X., Ma, Q.W., Eatock Taylor, R., 1998. Numerical simulation of sloshing waves in a 3D tank based on a finite element method. Applied Ocean Research 20,337-355.

Xue, M.A., Lin, P., 2011. Numerical study of ring baffle effects on reducing violent liquid sloshing. Computers \& Fluids 52, 116-129. 


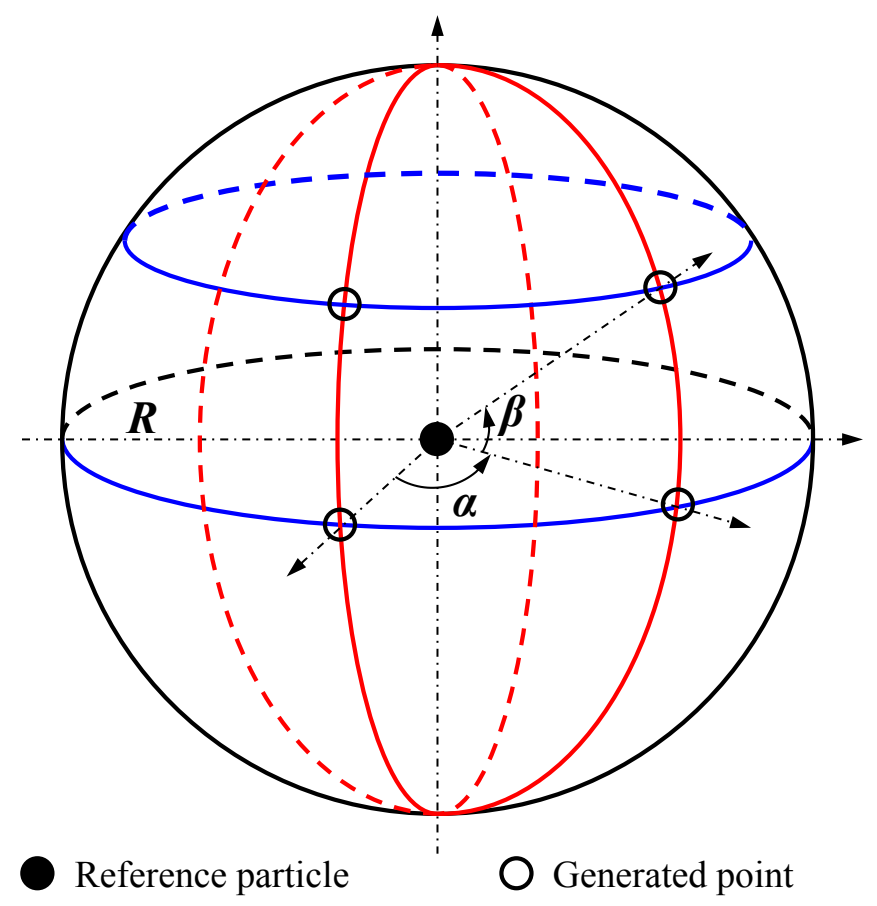

Fig. 1. Schematic view of 3D free surface recognition scheme

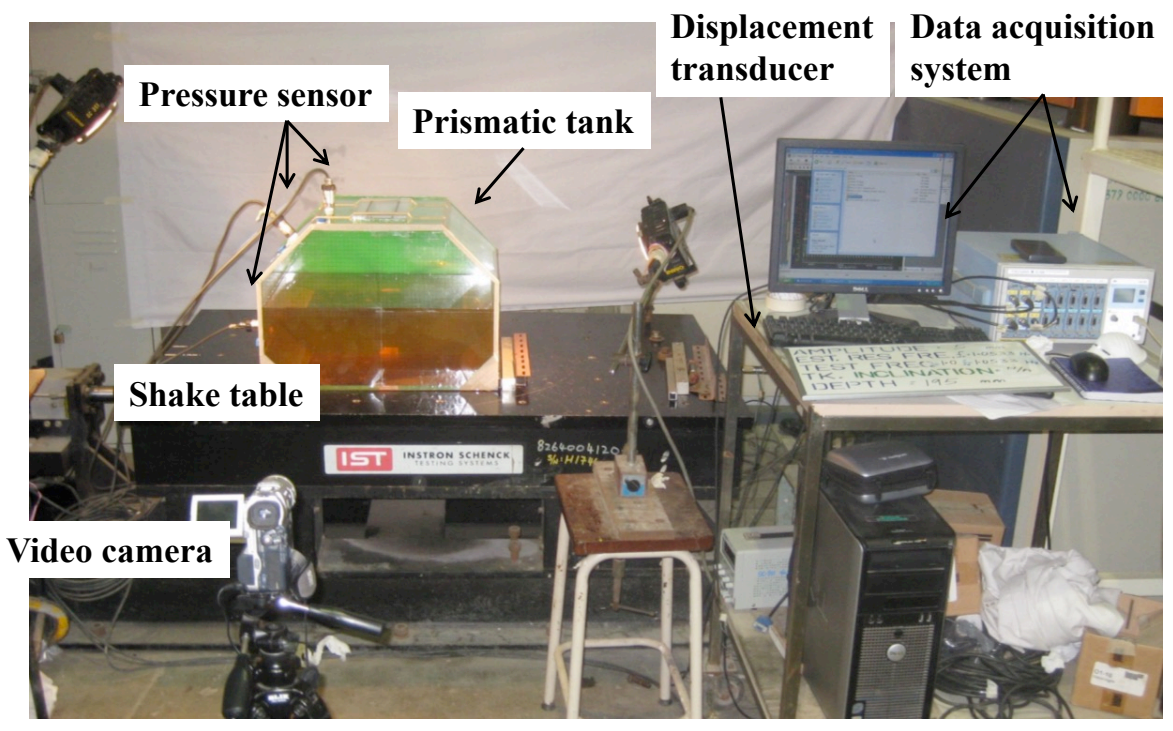

Fig. 2. Water sloshing on translational shake table 


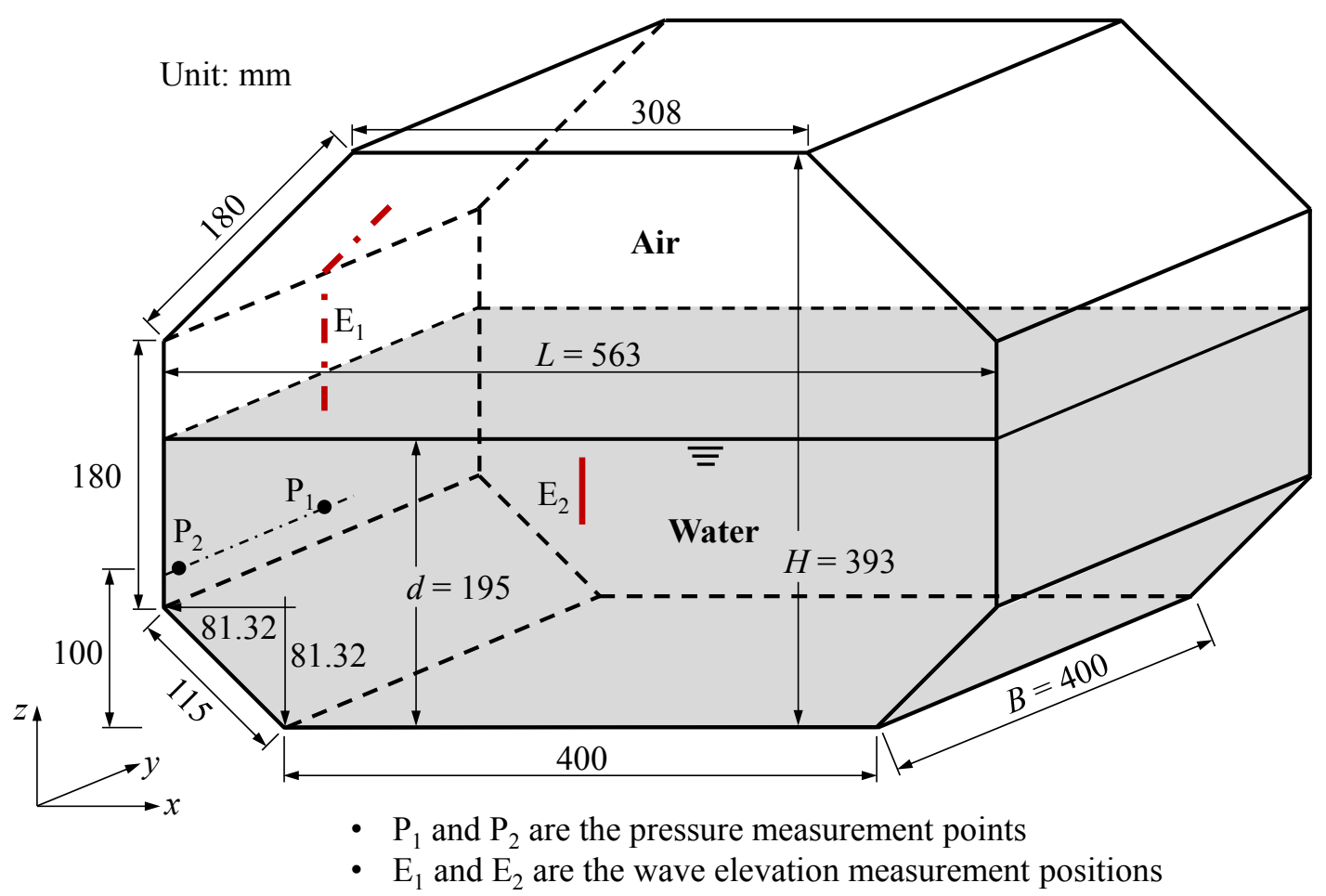

Fig. 3. Geometric dimensions of water tank used in sloshing experiments

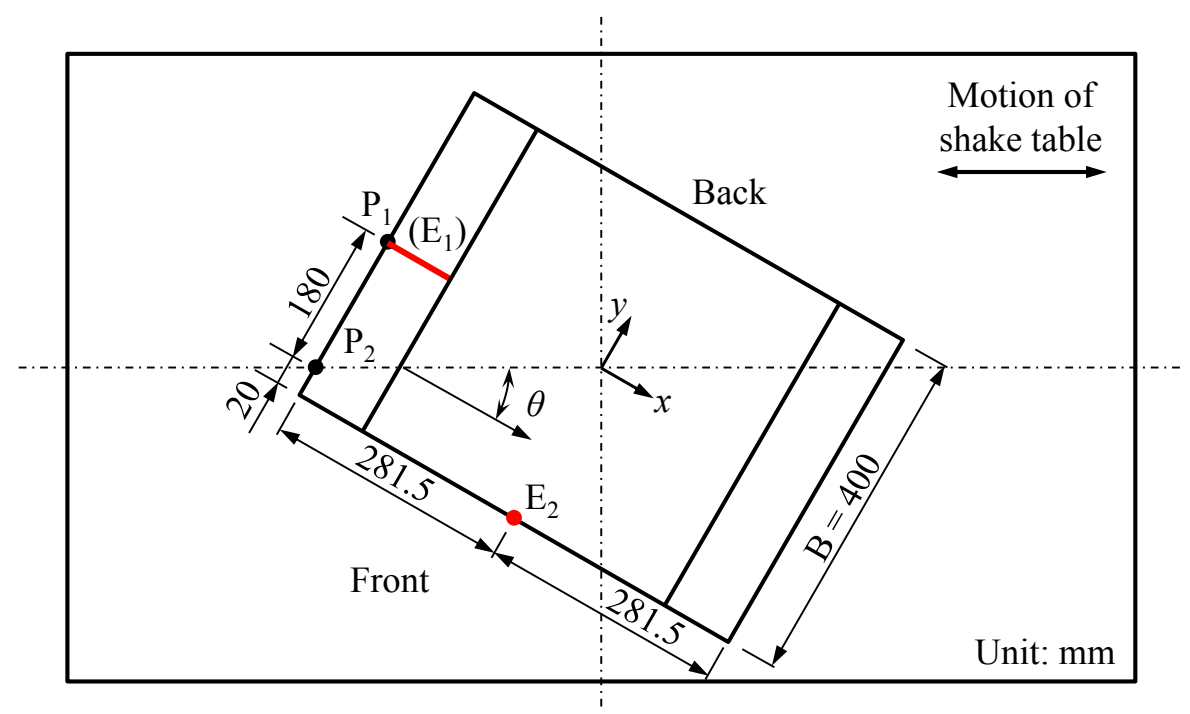

Fig. 4. Top view of 3D experiments on a translational shake table 


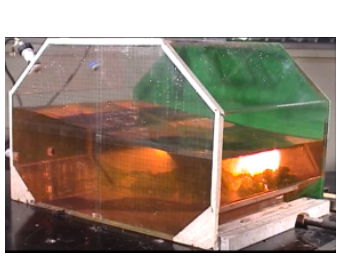

$t=8.02 \mathrm{~s}$

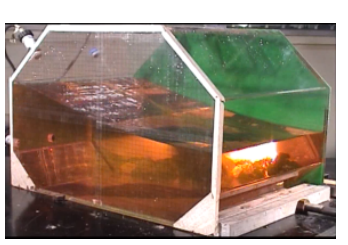

$t=9.92 \mathrm{~s}$

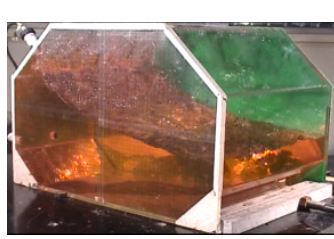

$t=13.72 \mathrm{~s}$
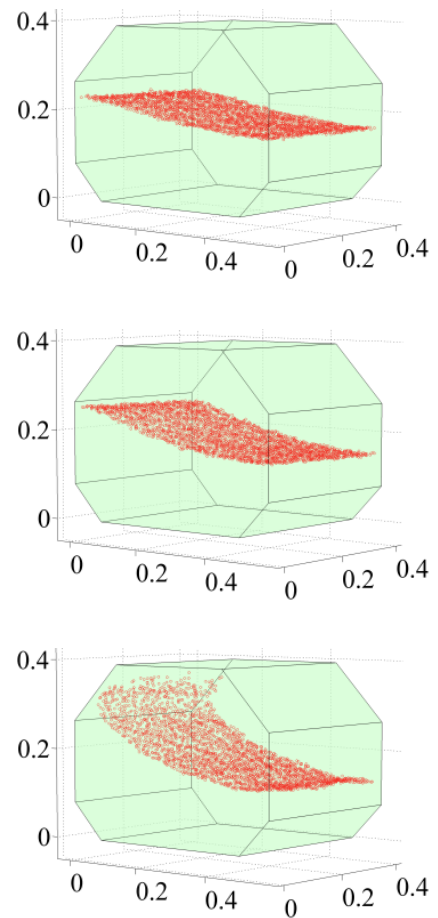

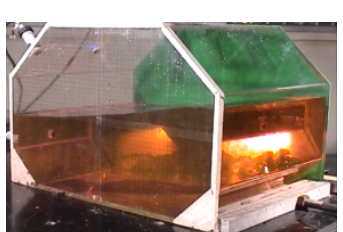

$t=8.52 \mathrm{~s}$

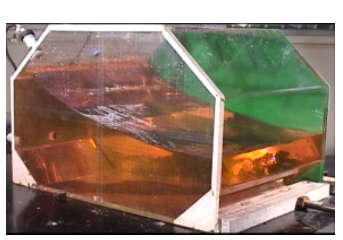

$t=11.82 \mathrm{~s}$

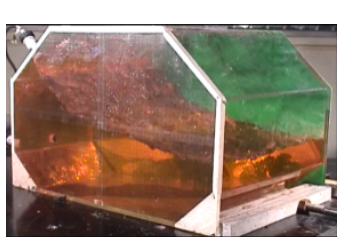

$t=14.675 \mathrm{~s}$
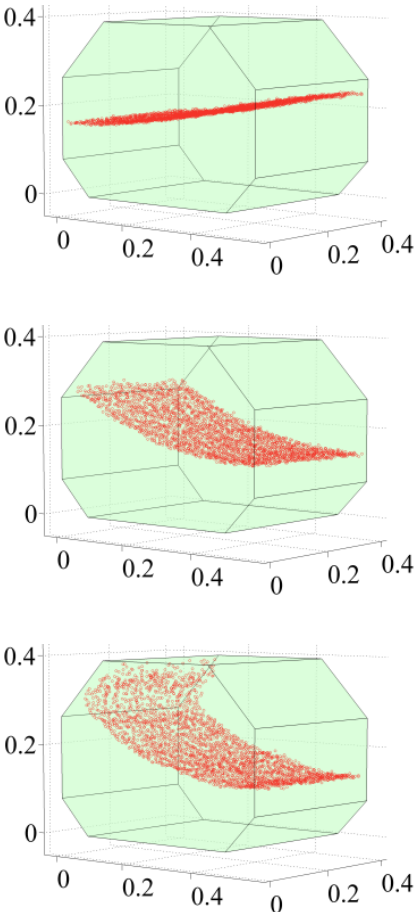

Fig. 5. Water sloshing under translational excitation $\left(\omega=\omega_{L 0}\right)$ : CPM result of sloshing waves in comparison with experimental results

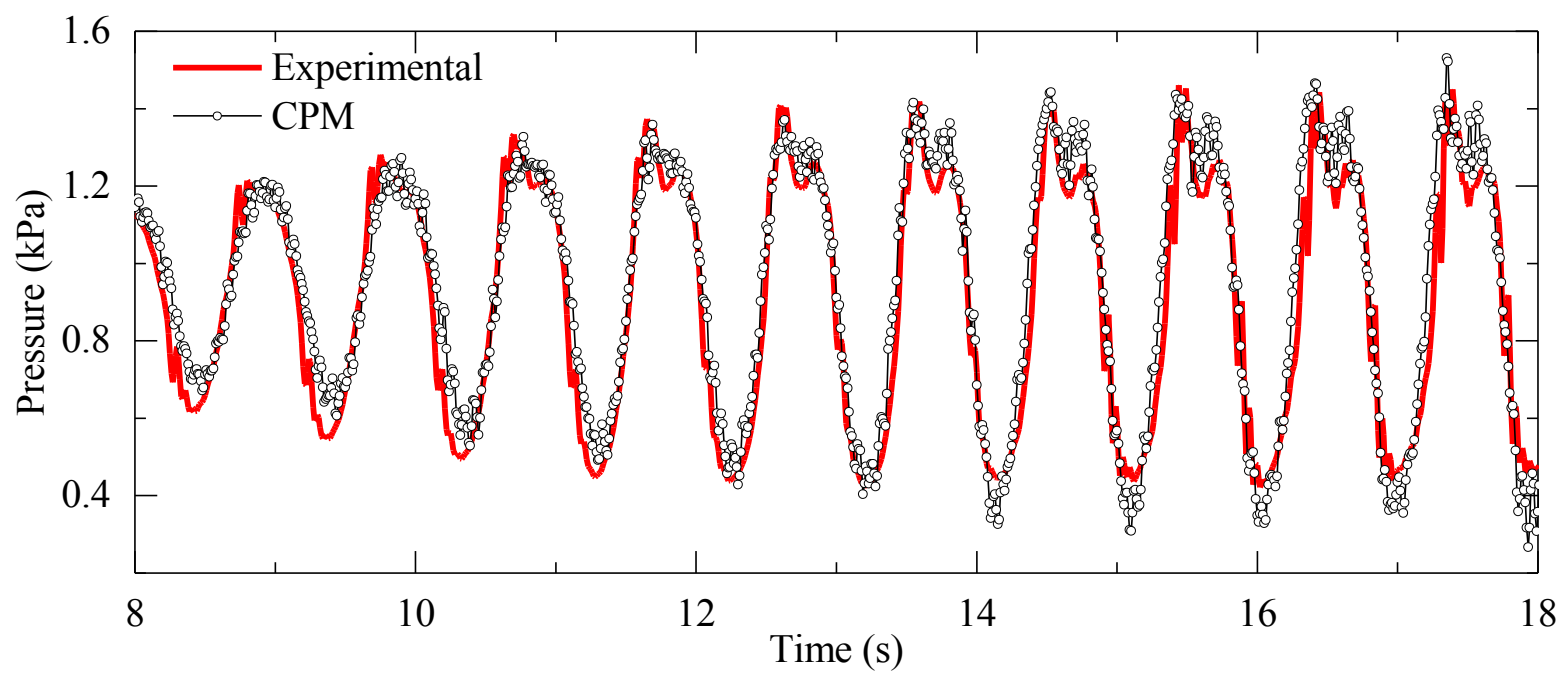

Fig. 6. Water sloshing under translational excitation $\left(\omega=\omega_{L 0}\right)$ : CPM result of pressure history at point $\mathrm{P}_{1}$ in comparison with experimental result 

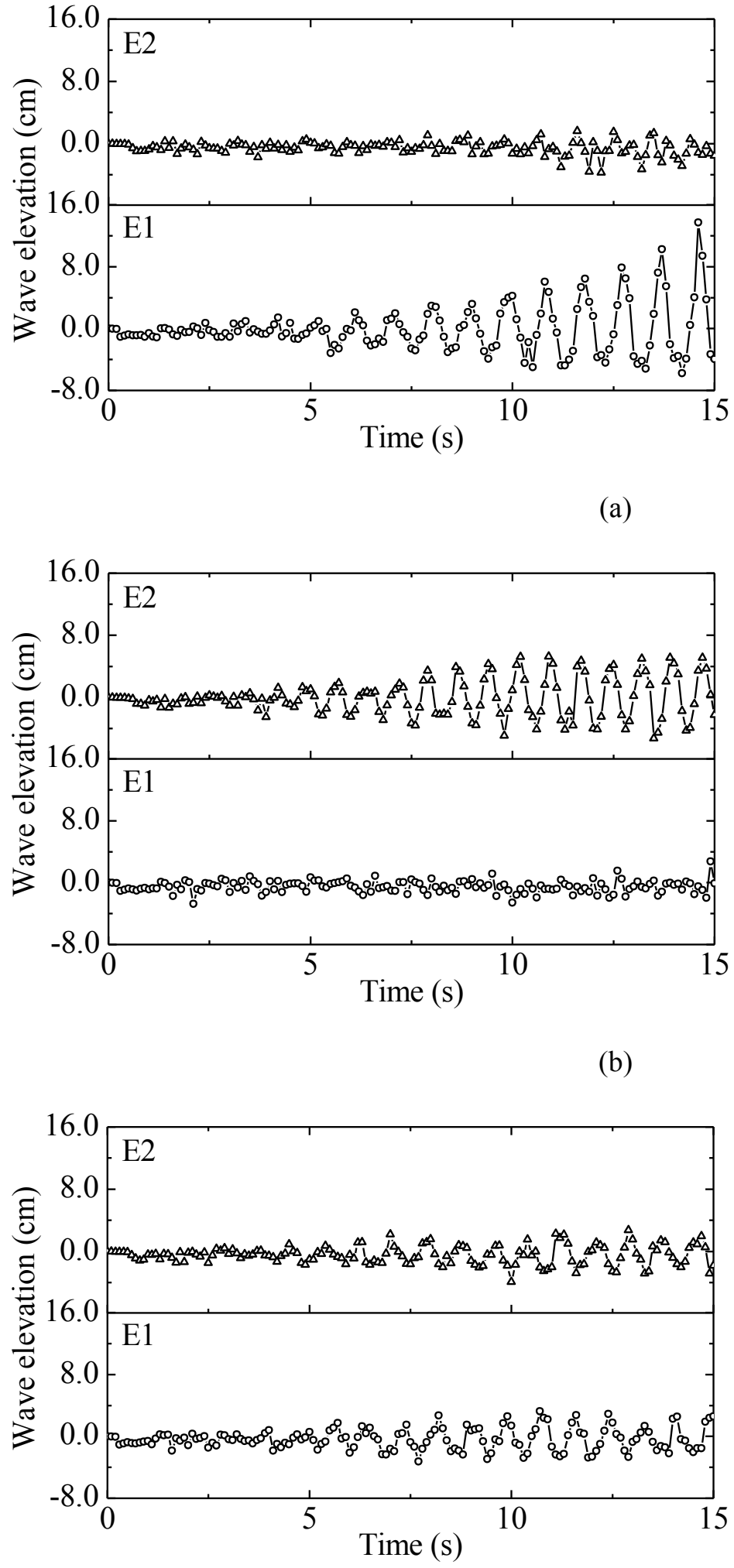

(a)

(b)
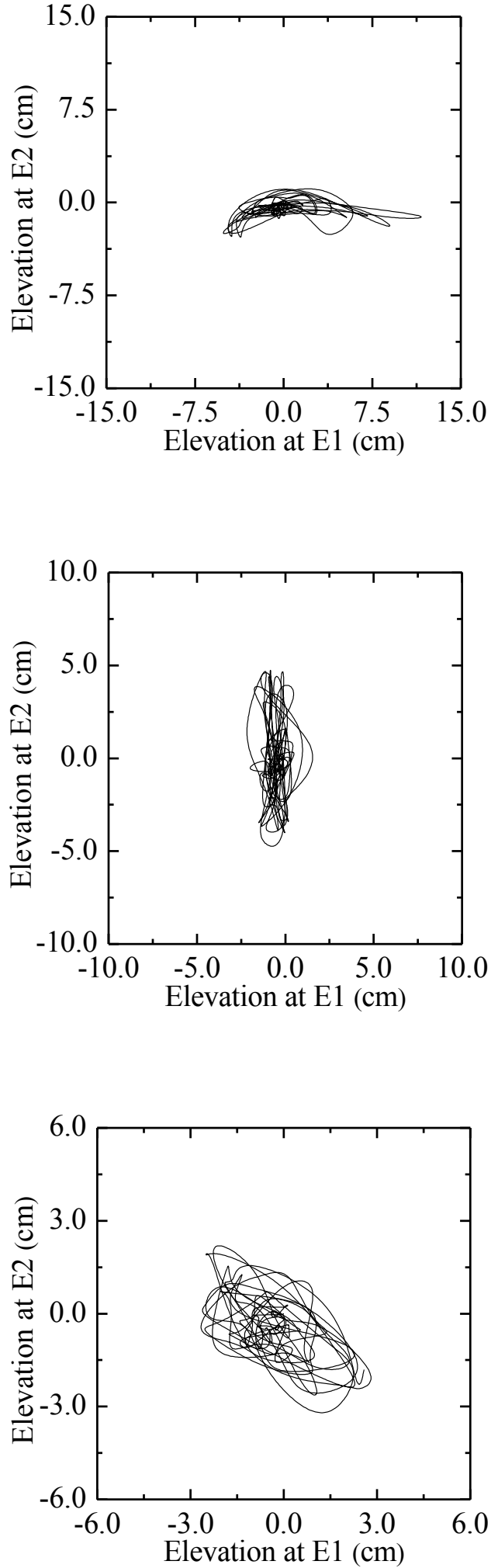

(c)

Fig. 7. Wave elevations at position $E_{1}$ and $E_{2}$ for typical cases of sloshing under translational excitations: (a) length-dominant wave with $\omega=\omega_{L 0}(6.618 \mathrm{rad} / \mathrm{s})$, (b) breadth-dominant wave with $\omega$ $=\omega_{B 0}(8.364 \mathrm{rad} / \mathrm{s})$ and (c) nearly-diagonal wave with $\omega=7.445 \mathrm{rad} / \mathrm{s}$ (between $\omega_{L 0}$ and $\left.\omega_{B 0}\right)$ 


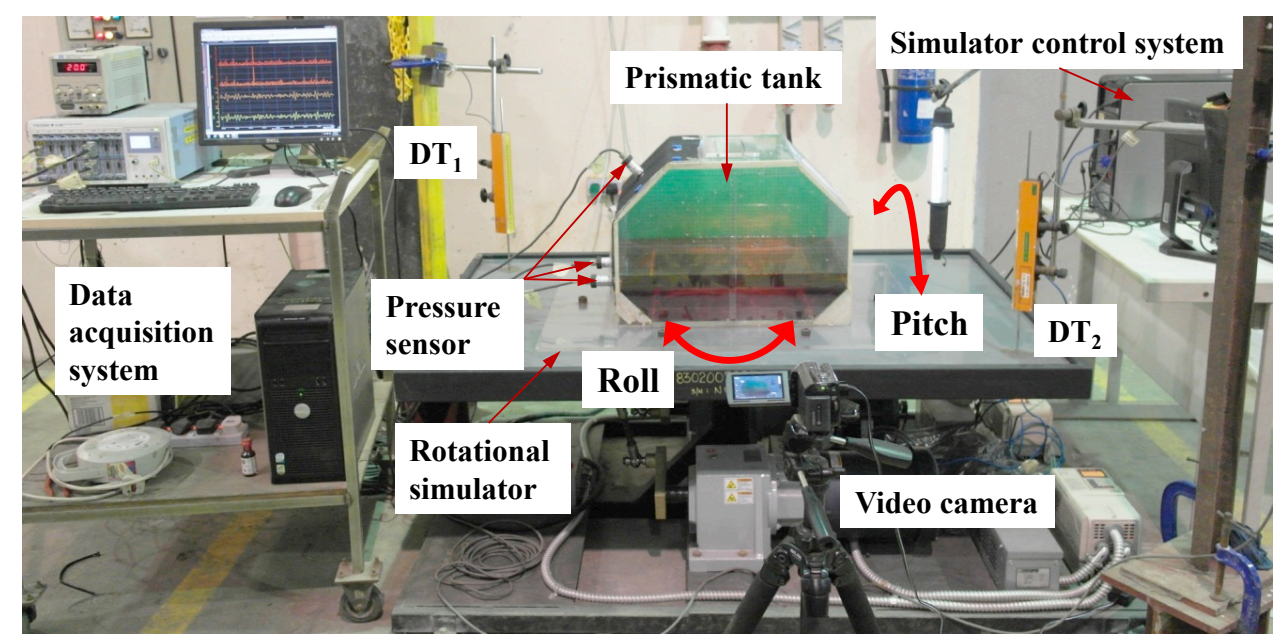

Fig. 8. Water sloshing on rotational simulator

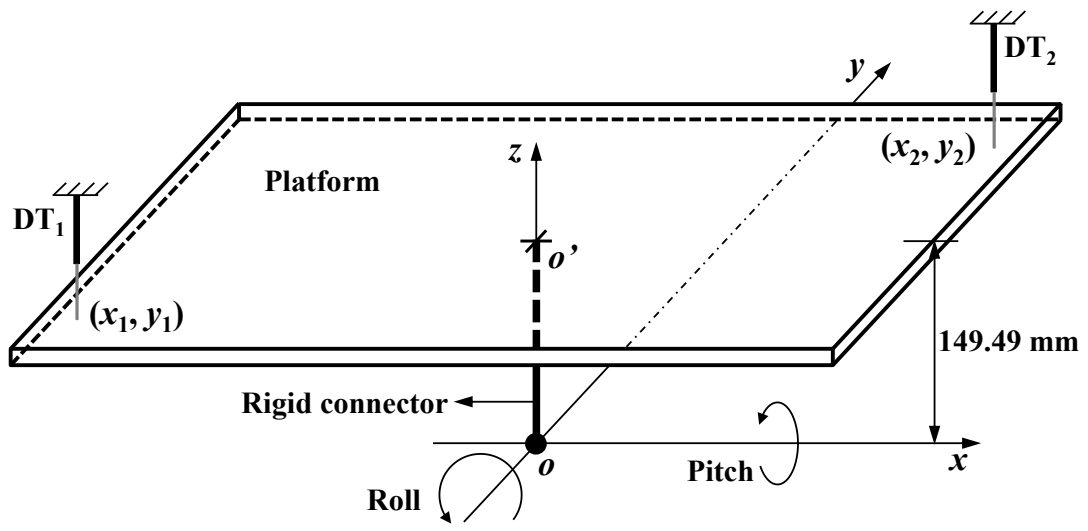

Fig. 9. Schematic view of the rotational simulator

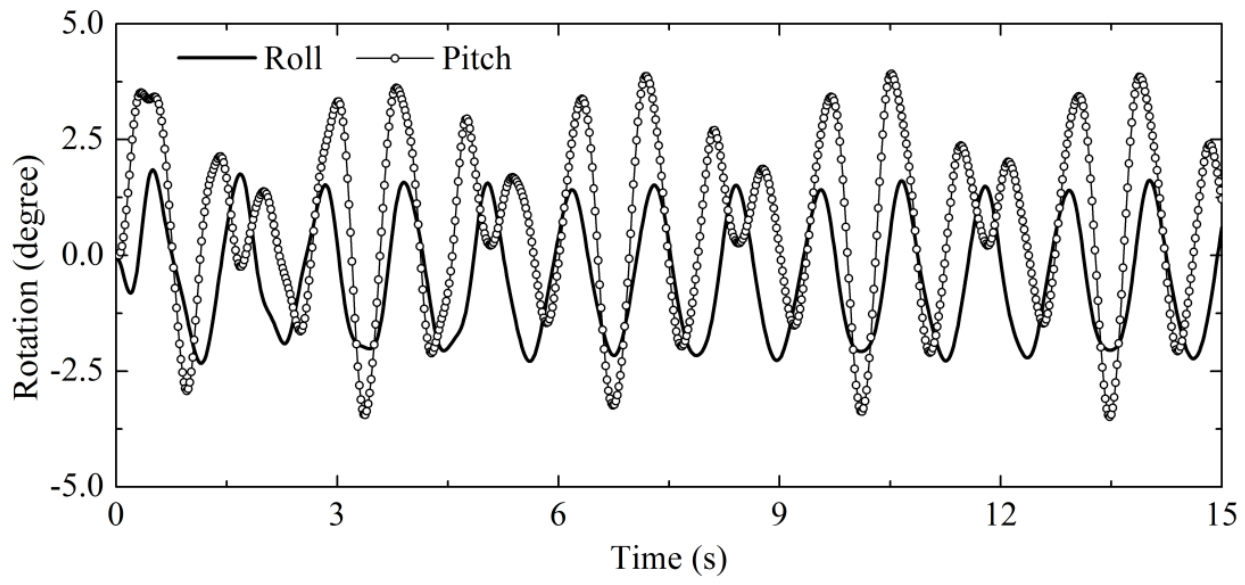

Fig. 10. Water sloshing under resonant rotational excitation: roll and pitch angles 

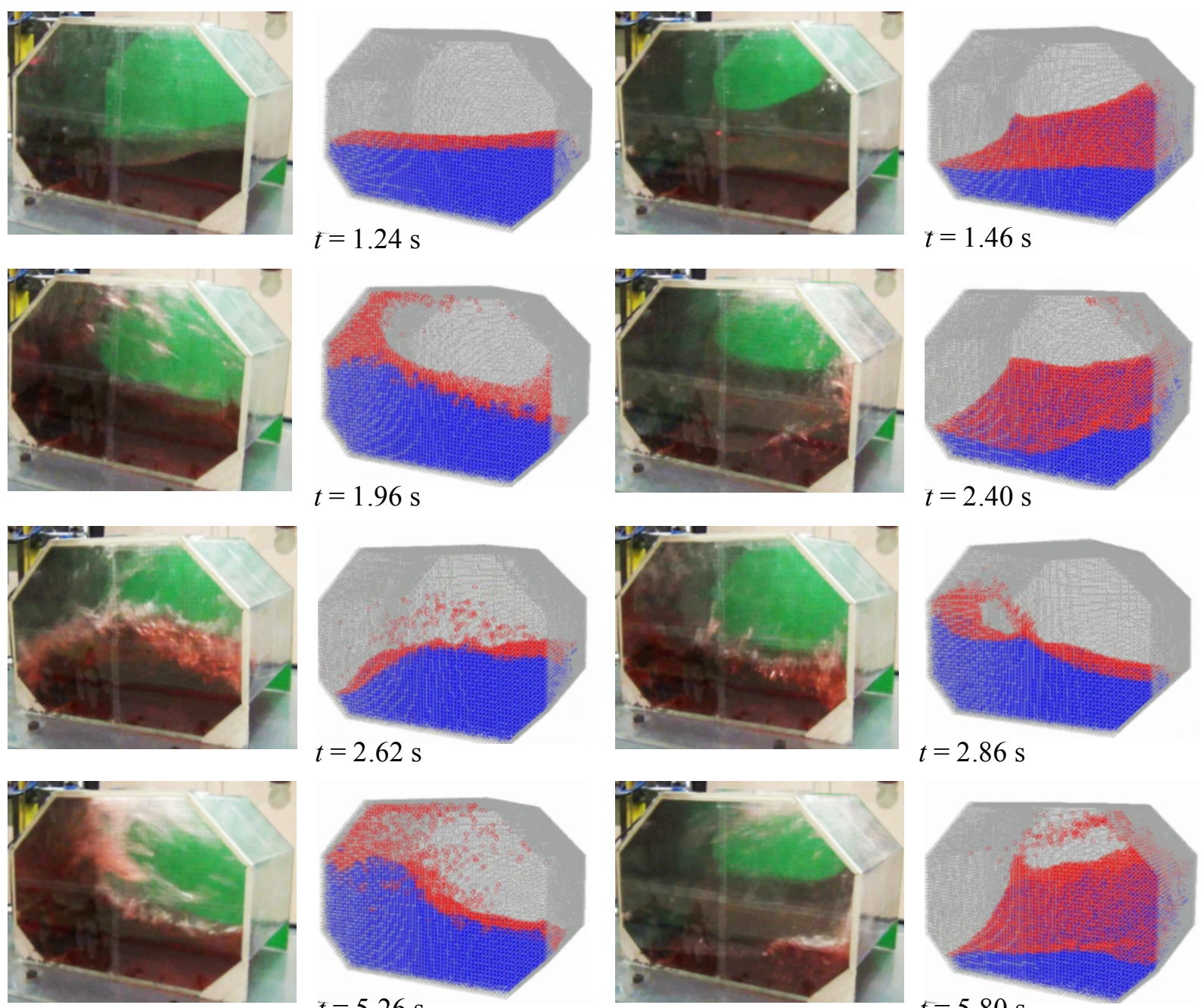

$t=2.86 \mathrm{~s}$
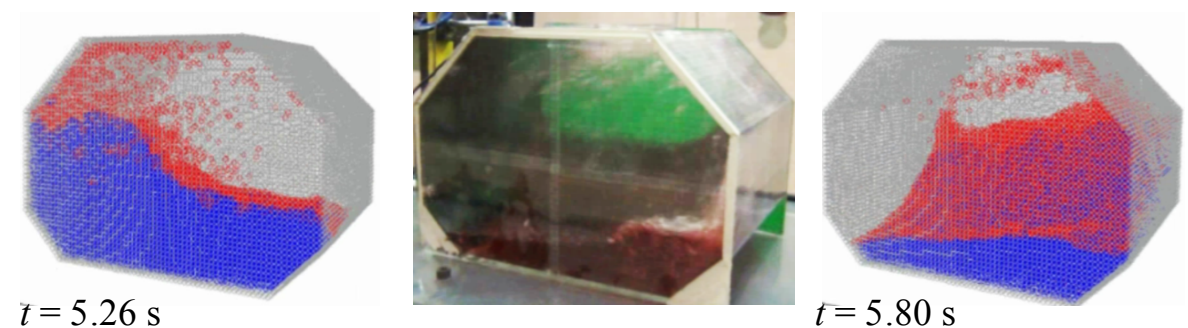

Fig. 11. Water sloshing under resonant rotational excitation: wave profiles at typical time instants

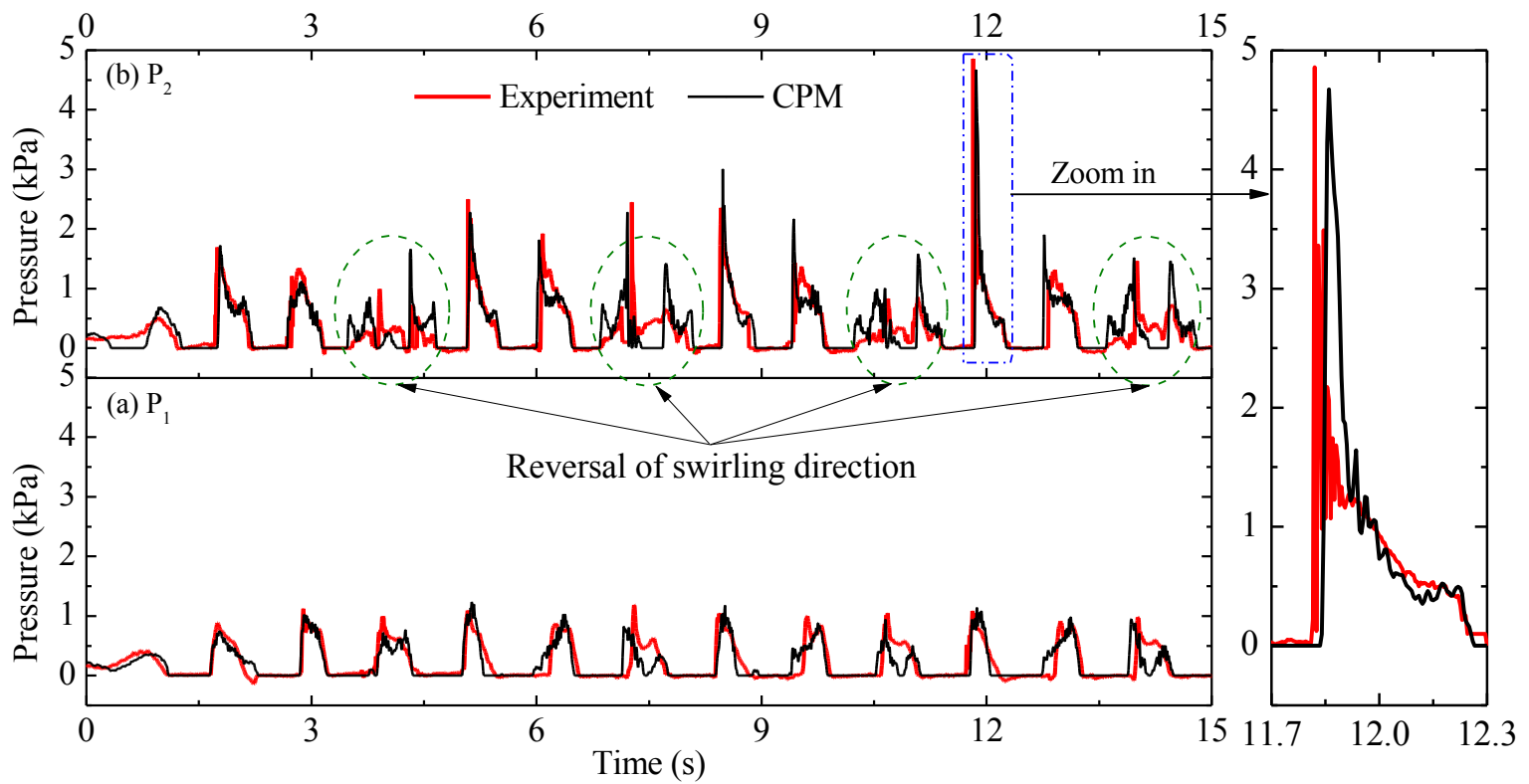

Fig. 12. Water sloshing under resonant rotational excitation: pressure histories at $P_{1}$ and $P_{2}$ 


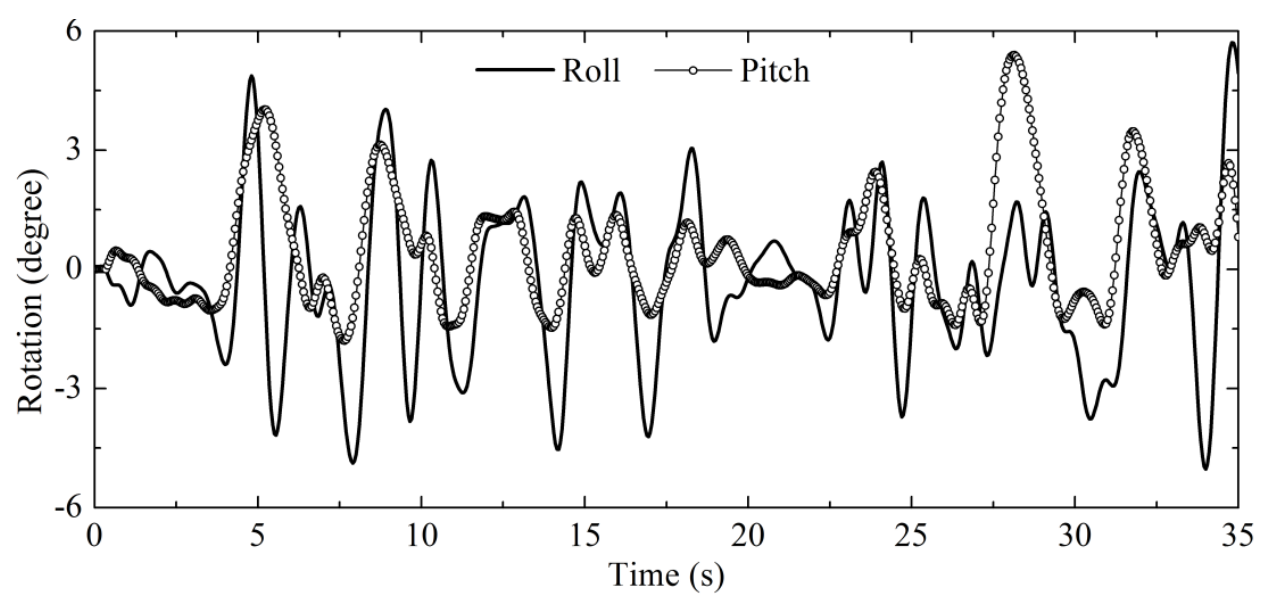

Fig. 13. Water sloshing under random rotational excitation: roll and pitch angles
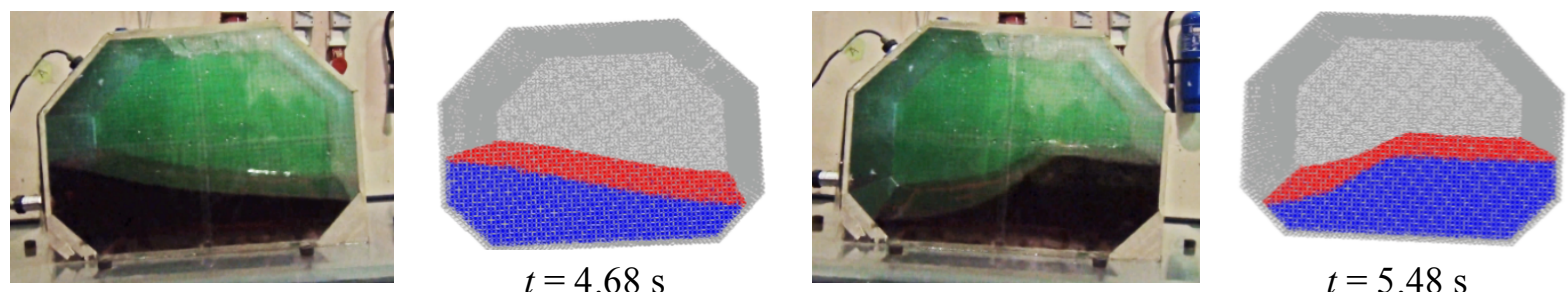

$t=4.68 \mathrm{~s}$

$t=5.48 \mathrm{~s}$
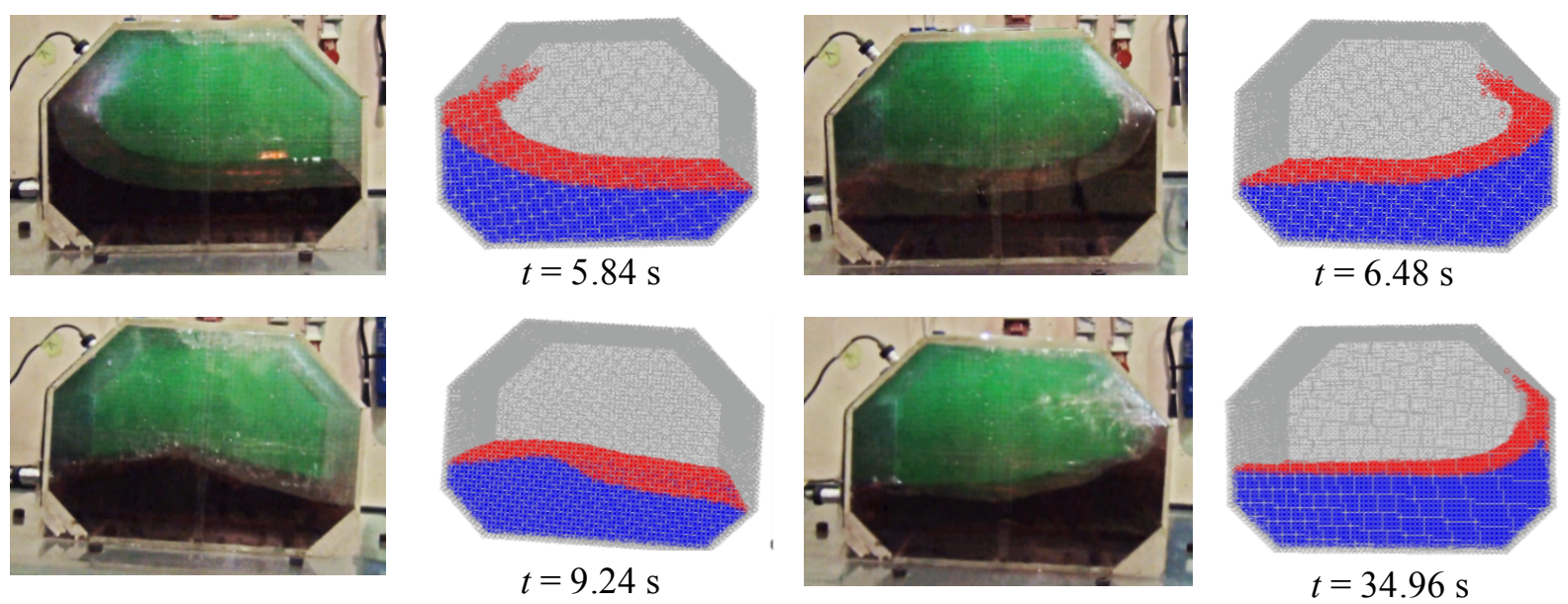

Fig. 14. Water sloshing under random rotational excitation: wave profiles at typical time instants 


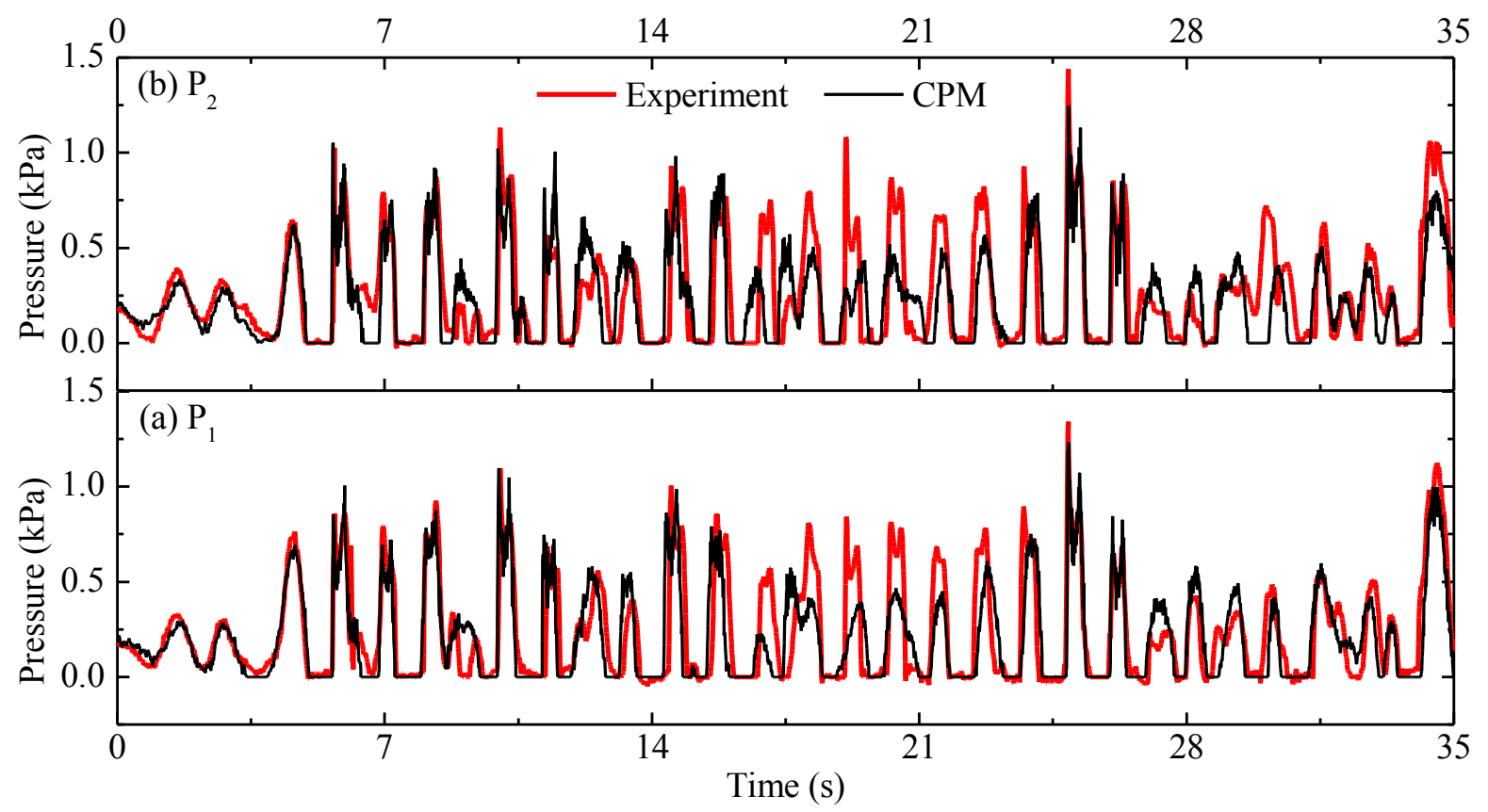

Fig. 15. Water sloshing under random rotational excitation: pressure histories at $\mathrm{P}_{1}$ and $\mathrm{P}_{2}$

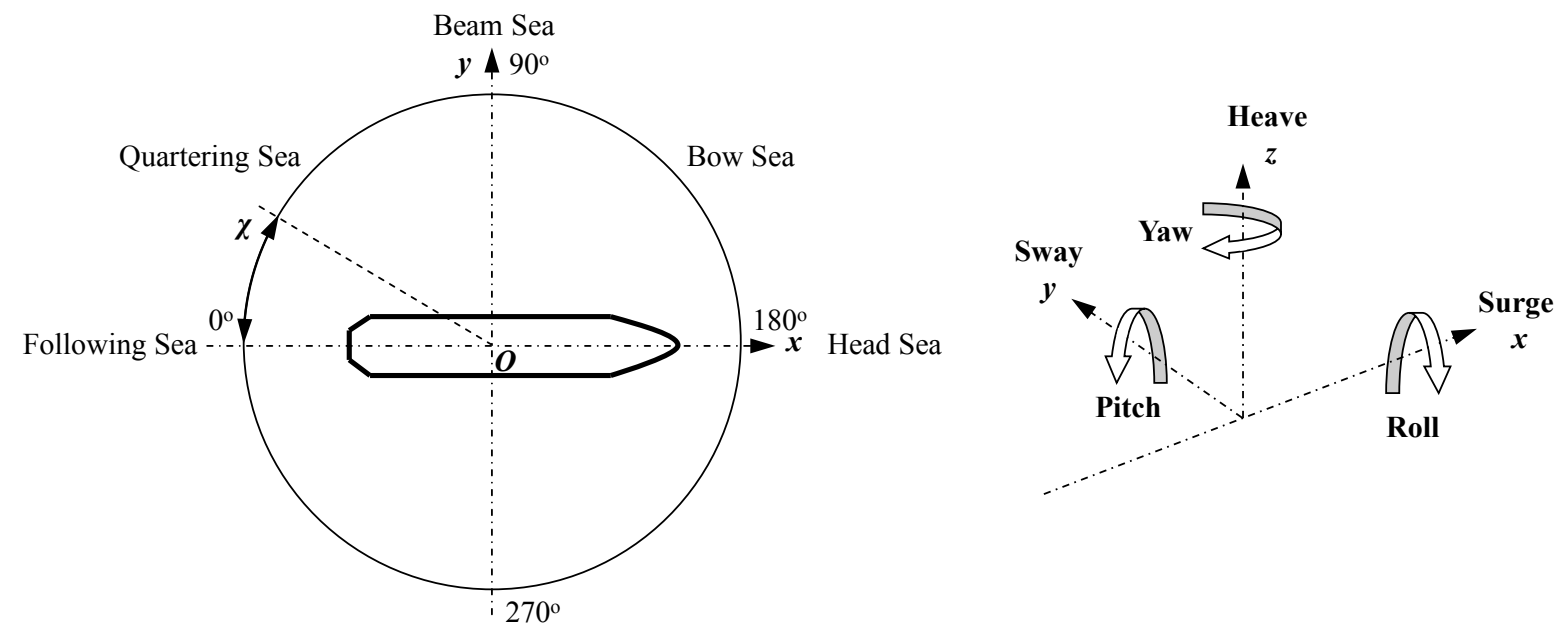

Fig. 16. Schematic diagram of a LNG ship and definition of encounter angle $\chi$ and different sailing conditions (Perez and Blanke, 2002) 


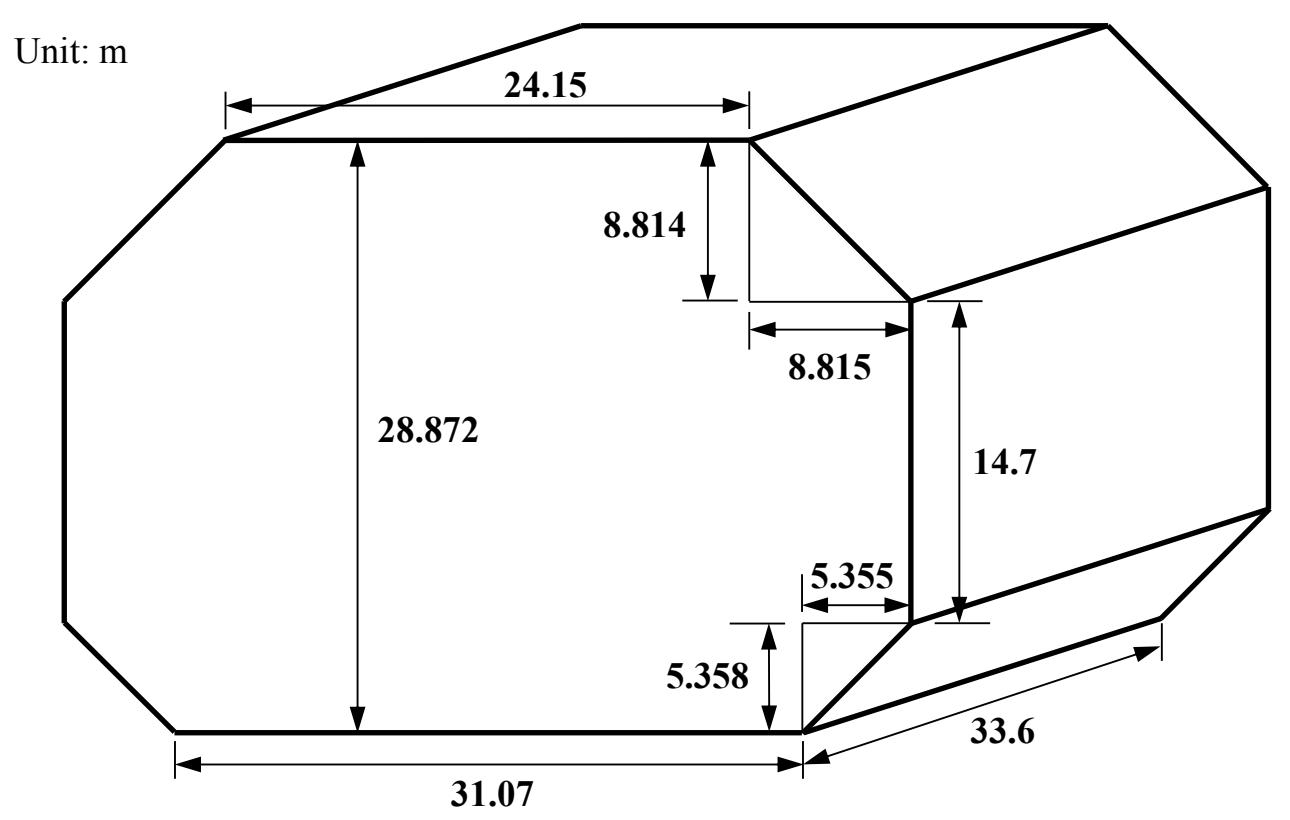

Fig. 17. Dimensions of a real LNG tank

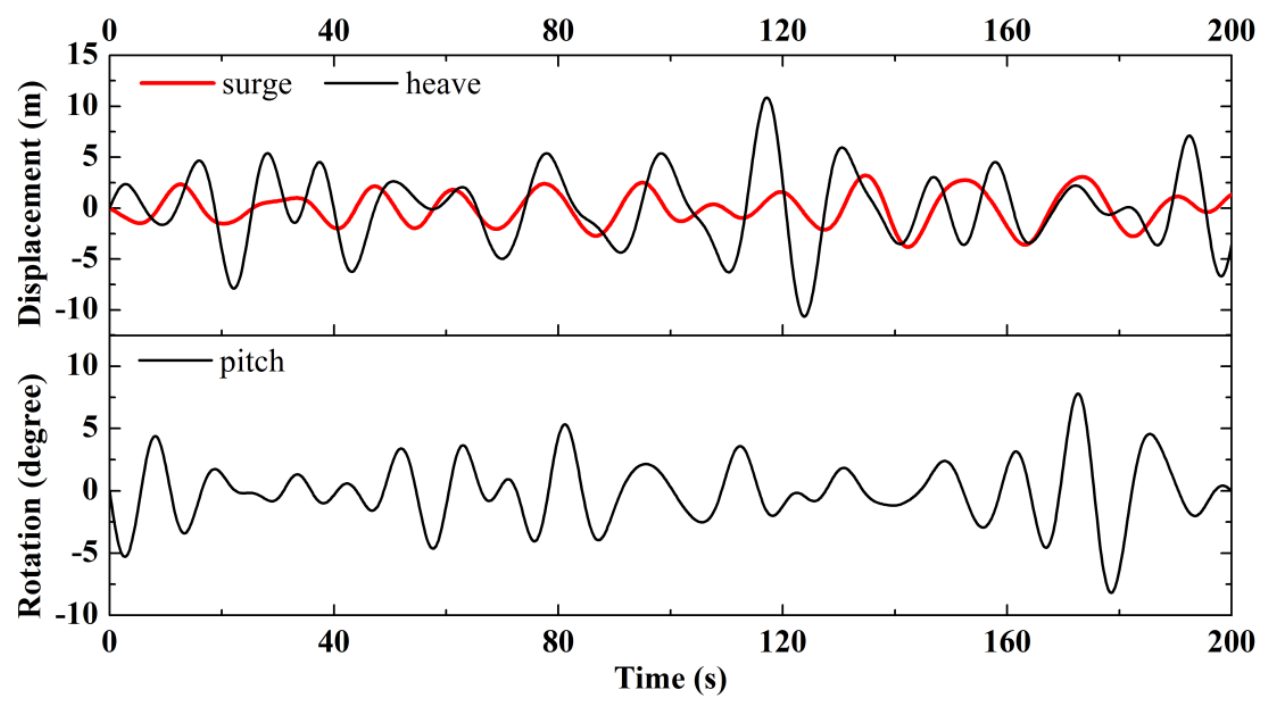

Fig. 18. Ship motions in Head Sea 

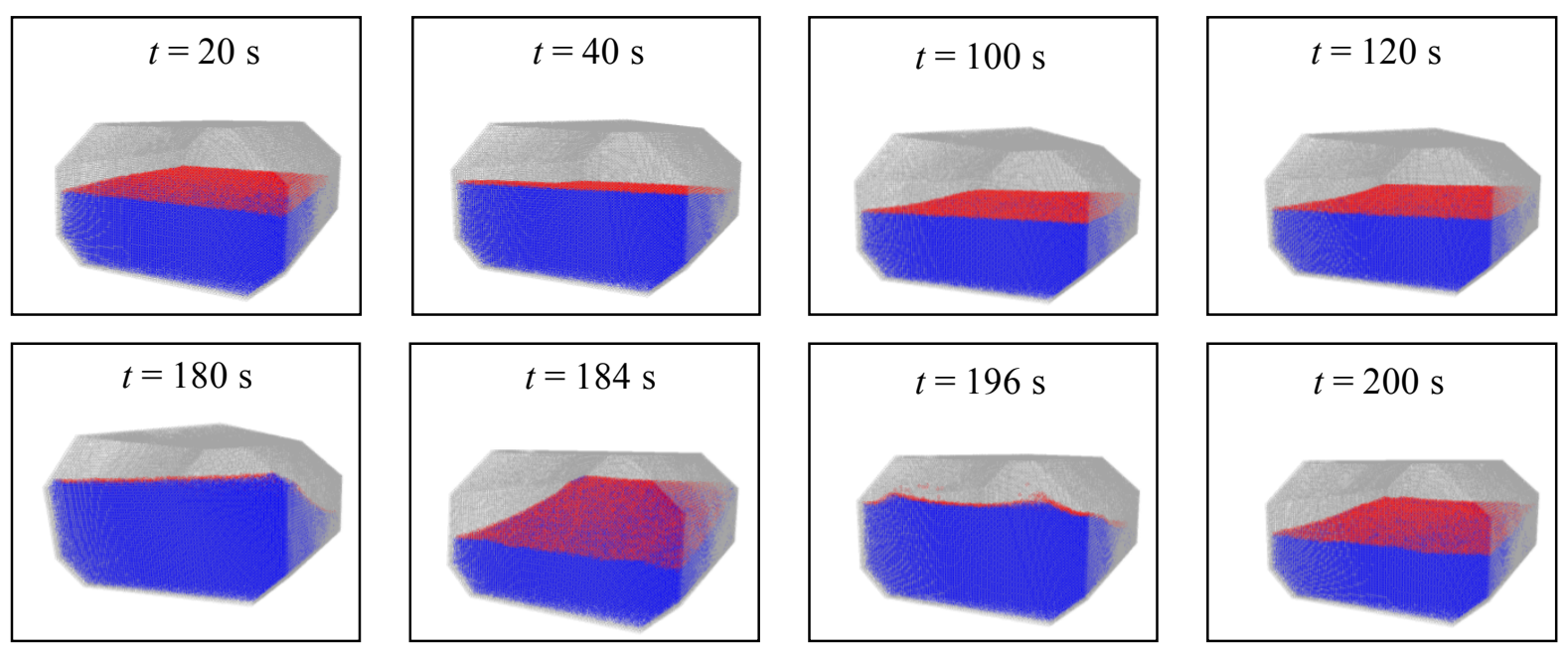

Fig. 19. Wave profiles of LNG sloshing in Head Sea

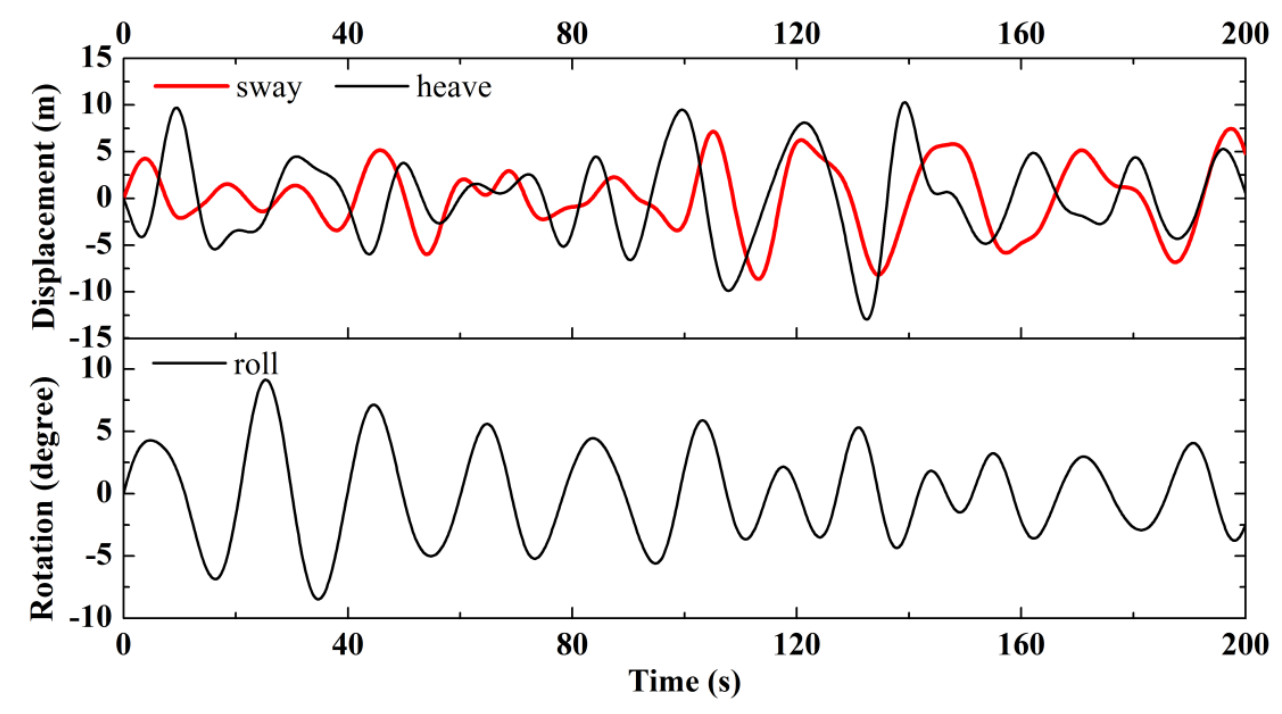

Fig. 20. Ship motion in Beam Sea
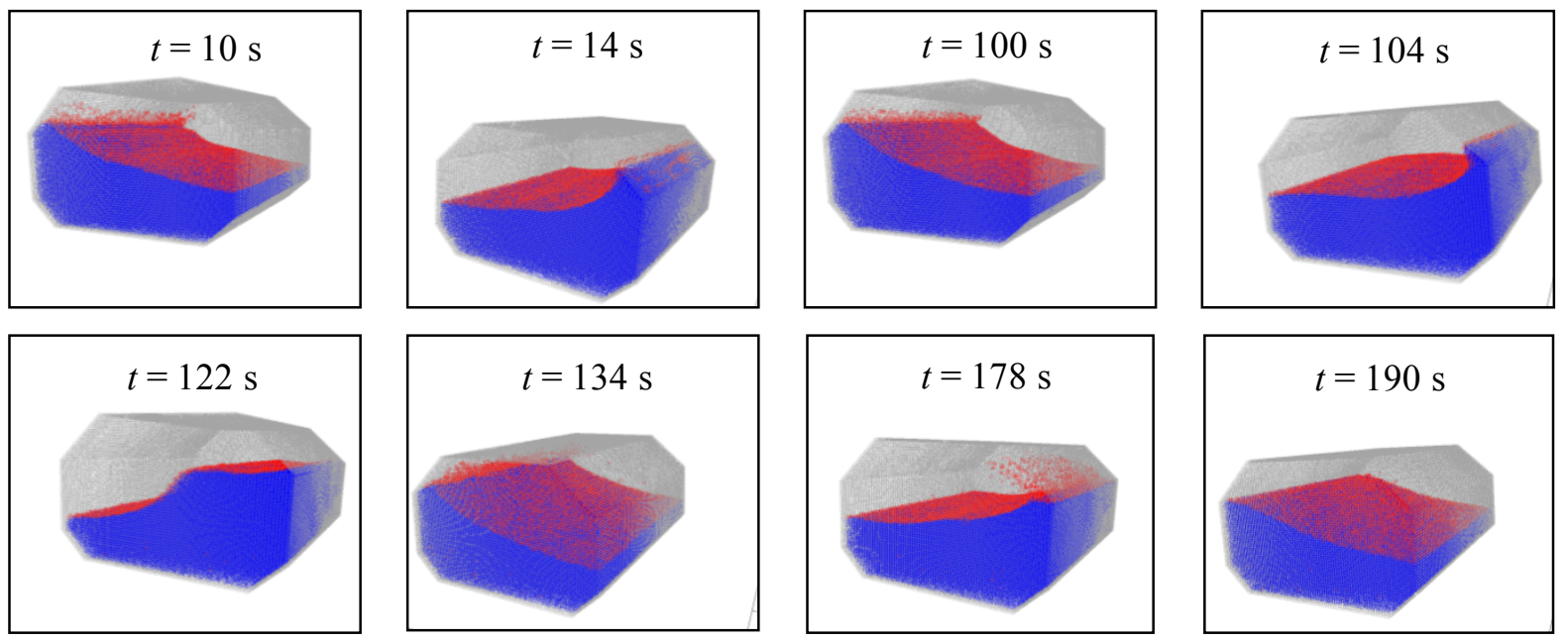

Fig. 21. Wave profiles of LNG sloshing in Beam Sea 


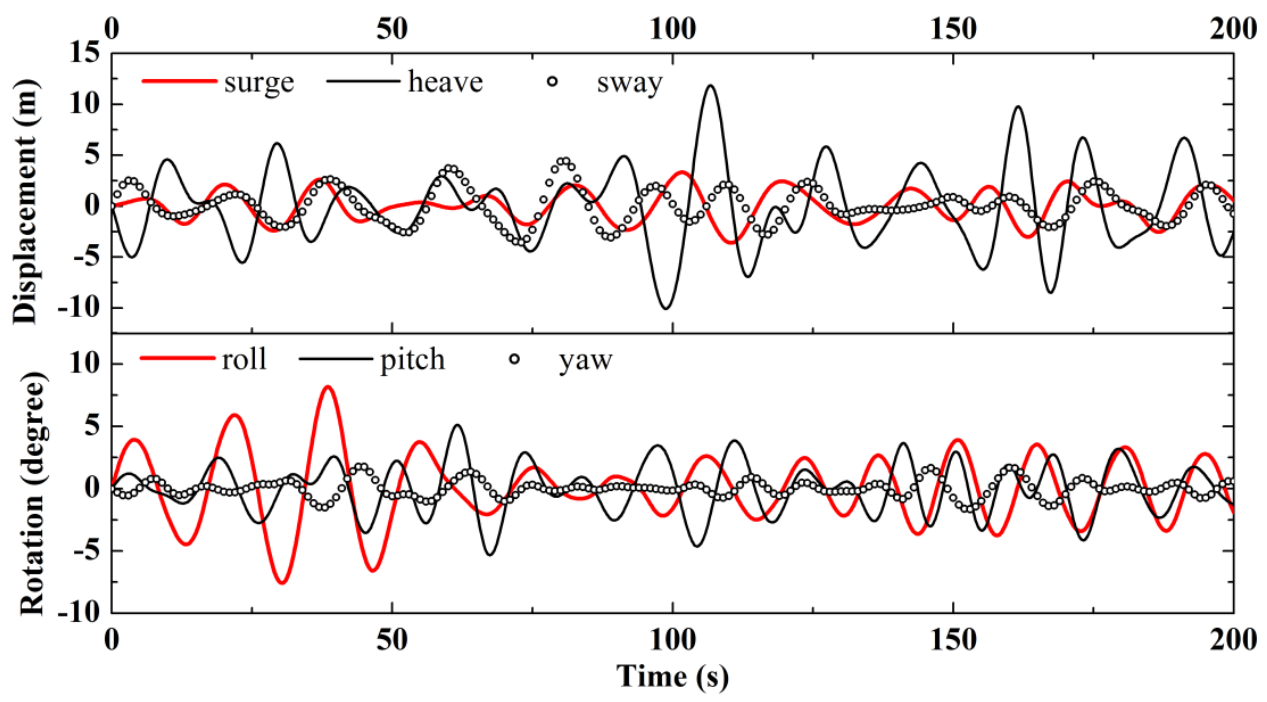

Fig. 22. Ship motions in Bow Sea 135 degrees
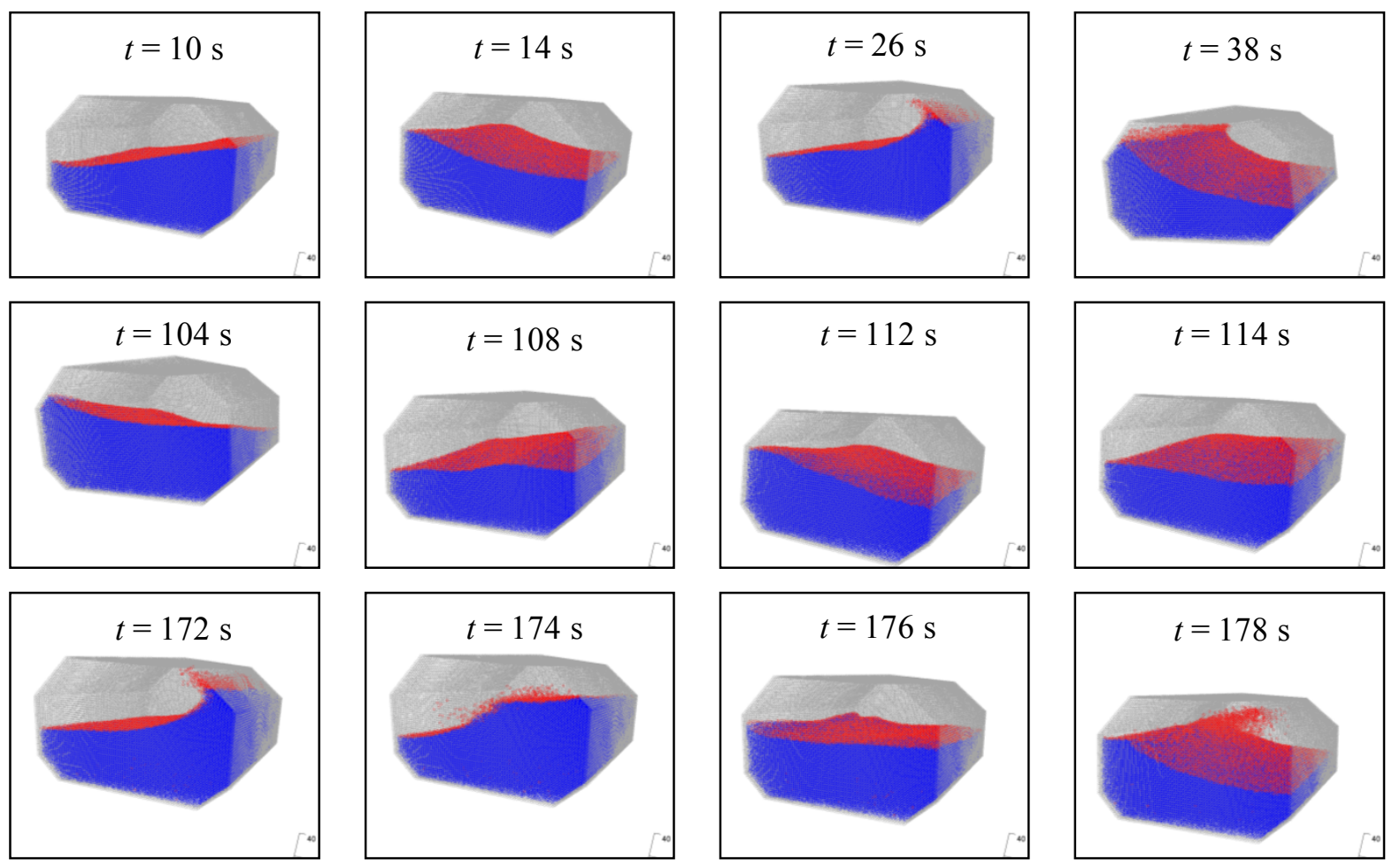

Fig. 23. Wave profiles of LNG sloshing in Bow Sea 135 degrees 